\title{
Reprogramming Müller glia via in vivo cell fusion regenerates murine photoreceptors
}

\author{
Daniela Sanges, ${ }^{1}$ Giacoma Simonte, ${ }^{1}$ Umberto Di Vicino, ${ }^{1}$ Neus Romo, ${ }^{1}$ Isabel Pinilla, ${ }^{2}$ Marta Nicolás, ${ }^{3}$ and Maria Pia Cosma ${ }^{1,4}$ \\ ${ }^{1}$ Center for Genomic Regulation (CRG), The Barcelona Institute of Science and Technology, Barcelona, Spain. ${ }^{2}$ Aragon Health Science Institute and University of Zaragoza, Zaragoza, Spain. \\ ${ }^{3}$ Ferrer International SA, Barcelona, Spain. ${ }^{4}$ Institució Catalana de Recerca i Estudis Avançats (ICREA), Barcelona, Spain.
}

\begin{abstract}
Vision impairments and blindness caused by retinitis pigmentosa result from severe neurodegeneration that leads to a loss of photoreceptors, the specialized light-sensitive neurons that enable vision. Although the mammalian nervous system is unable to replace neurons lost due to degeneration, therapeutic approaches to reprogram resident glial cells to replace retinal neurons have been proposed. Here, we demonstrate that retinal Müller glia can be reprogrammed in vivo into retinal precursors that then differentiate into photoreceptors. We transplanted hematopoietic stem and progenitor cells (HSPCs) into retinas affected by photoreceptor degeneration and observed spontaneous cell fusion events between Müller glia and the transplanted cells. Activation of Wnt signaling in the transplanted HSPCs enhanced survival and proliferation of MüllerHSPC hybrids as well as their reprogramming into intermediate photoreceptor precursors. This suggests that Wnt signaling drives the reprogrammed cells toward a photoreceptor progenitor fate. Finally, Müller-HSPC hybrids differentiated into photoreceptors. Transplantation of HSPCs with activated Wnt functionally rescued the retinal degeneration phenotype in rd10 mice, a model for inherited retinitis pigmentosa. Together, these results suggest that photoreceptors can be generated by reprogramming Müller glia and that this approach may have potential as a strategy for reversing retinal degeneration.
\end{abstract}

\section{Introduction}

Despite long-held dogma on the impossibility of changing the identity of differentiated cells, the expression of specific transcription factors, cell-cell fusion, and nuclear transfer experiments has shown the feasibility of reprogramming cellular identity toward both pluripotency and unrelated cell fates (1-5). Neurons are still considered among the most immutable cell types; nevertheless, recent studies have demonstrated that resident nonneuronal cells can be reprogrammed into induced neurons in vivo (6). This suggests that self-repair of nervous tissue can be promoted by inducing the fate conversion of resident cells into the desired cell type. Glial cells show the potential to divide and are very abundant in nervous tissue; therefore, they have been proposed as the ideal candidate cell type to generate new neurons. Glial cells can be turned into morphologically identifiable neurons in adult striatum upon expression of a cocktail of transcription factors (7) or just by the overexpression of Sox2, passing through an intermediate neuroblast stage (8).

In vivo conversion of glial cells into neurons represents a self-healing mechanism to regenerate nervous tissue in some species. Müller glia (MG) is the major glial cell type in the retina,

Conflict of interest: D. Sanges and M.P. Cosma hold a patent (PCT/EP2012/065327) related to the technology described in this manuscript that was licensed to Ferrer International SA. M.P. Cosma received licensing fees from Ferrer International SA for a different patent (PCT/EP2009/051512). D. Sanges and M.P. Cosma received research grants from Ferrer International. M.N. Farrés is directly employed at Ferrer International, but has no other financial interest.

Submitted: October 22, 2015; Accepted: May 24, 2016.

Reference information: / Clin Invest. 2016;126(8):3104-3116. doi:10.1172/JCI85193. is common to all vertebrates, and normally contributes to retinal structure and homeostasis (9). After an injury, zebrafish MG dedifferentiates and generates proliferating multipotent neural progenitors, which in turn exit the cell cycle and redifferentiate, replacing all lost retinal cell types with sight restoration (10-12). In mammals, MG normally responds to damage by becoming reactive and undergoing gliosis $(13,14)$. However, some studies have indicated that a limited numbers of mouse and rat MG can reenter the cell cycle and be converted into retinal neurons after retinal injury and upon stimulus induction or expression of specific factors (15-18). These early studies made some pioneering observations; however, direct conversion of MG into neurons can be definitely concluded only with lineage-tracing experiments (19). Overall, the efficiency of the reported conversion was low or was not quantified, and importantly, electrophysiological functionality of the neurons obtained was not investigated. Nevertheless, these results suggest that the barrier that blocks $\mathrm{MG}$ reprogramming can also potentially be overcome in mammals.

Interestingly, the activation of Wnt/ $\beta$-catenin signaling can mediate proliferation of $\mathrm{MG}$ in the injured mammalian retina (16). Wnt/ $\beta$-catenin signaling strongly enhances the efficiency of reprogramming of somatic cells upon cell fusion with pluripotent stem cells $(20,21)$. Transplanted BM-derived cells (BMDCs) can fuse with somatic cells and generate hybrids, which in turn can differentiate in myocytes (22), hepatocytes $(23)$, neurons $(24,25)$, and other cell types in vivo $(26,27)$. We recently showed that, upon drug-induced ganglion cell (GC) damage, transplanted Wnt-activated hematopoietic stem and progenitor cells (HSPCs) can fuse with retinal cells. The newly formed hybrids undergo reprogramming and differentiate into ganglion and amacrine neurons (28). 
A

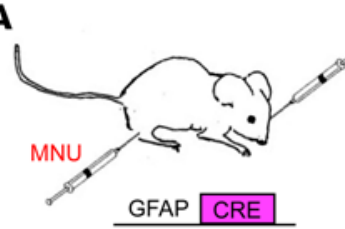

Recipient MG
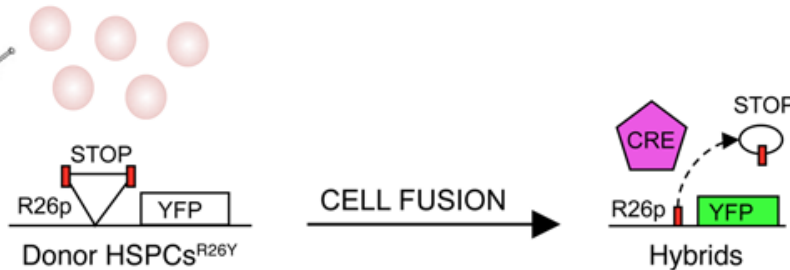

Hybrids
B

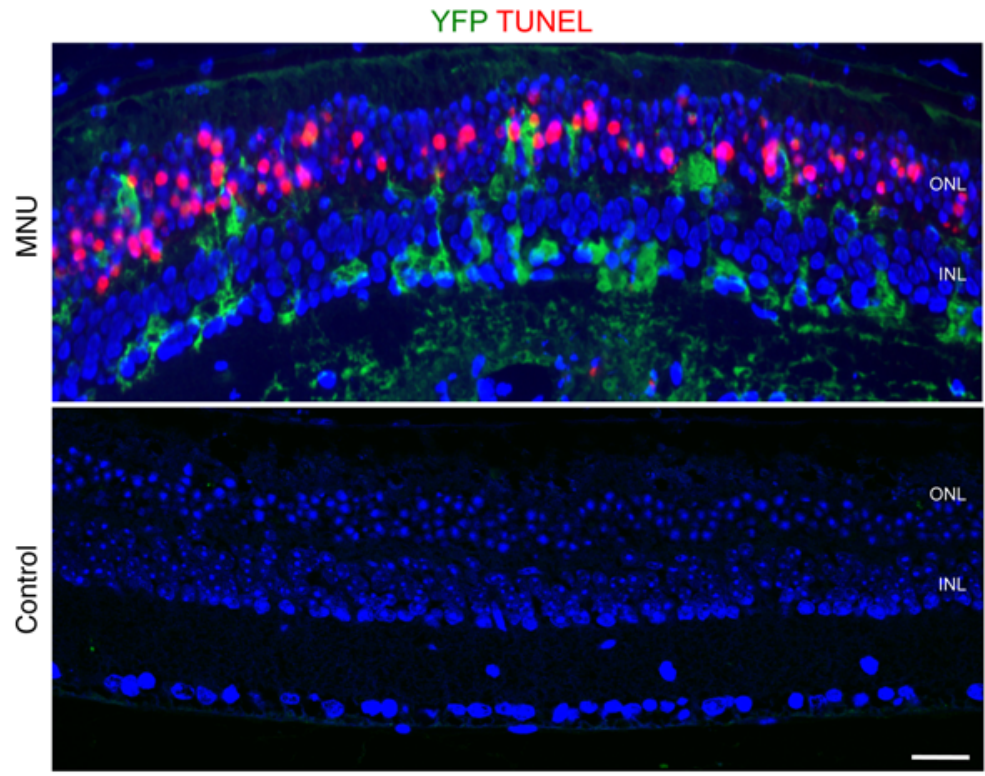

C

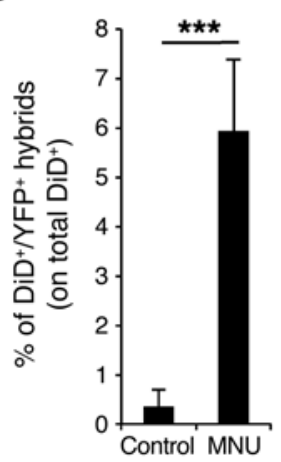

D

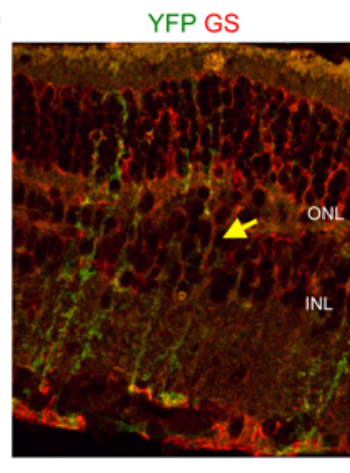

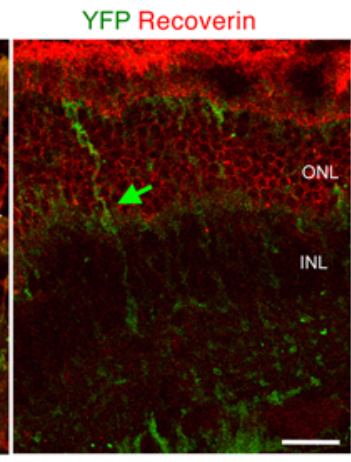

Figure 1. Transplanted HSPCs fuse with MG upon photoreceptor damage. (A) Schematic representation of the experimental plan. Cell fusion between HSPCs isolated from LoxPSTOP-LoxP-YFP donor mice (R26Y) and recipient Gfap-Cre MG cells leads to excision of the floxed stop codon and, in turn, to the expression of YFP. (B) Representative coimmunostaining of $\mathrm{YFP}^{+}$ hybrids (green) and TUNEL+ (red) apoptotic photoreceptors on retinal sections harvested from MNU-damaged or healthy (control) Gfap-Cre eyes 12 hours after subretinal transplantation of $\mathrm{HSPCS}^{\mathrm{R} 2 \mathrm{~V}}$. YFP ${ }^{+}$hybrids (green) derived from cell fusion are detected in MNU-damaged retinas, which show TUNEL ${ }^{+}$photoreceptors in the ONL, but not in the undamaged eyes (control). Nuclei were counterstained with DAPI (blue). Scale bar: $20 \mu \mathrm{m} . n=3$. (C) Statistical analysis of the percentage of $\mathrm{DiD}^{+} \mathrm{YFP} \mathrm{P}^{+}$hybrids evaluated on the total amount of DiD-labeled $\mathrm{HSPCS}^{\mathrm{R} 26 \mathrm{Y}}$ detected by FACS analysis in MNU-damaged or healthy (control) Gfap-Cre retinas 24 hours after transplantation. Data are represented as mean \pm SD of 3 independent experiments. $n=3 .{ }^{* *} P<0.0001$ by unpaired Student's $t$ test. (D) Representative immunostaining of $\mathrm{YFP}^{+}$hybrids (green) also positive for the MG marker (CS, red; yellow arrows) but not for the photoreceptor marker recoverin (red; green arrow) detected 24 hours after transplantation of HSPC $s^{\mathrm{R} 26 \mathrm{Y}}$ in MNU-damaged Gfap-Cre retinas. Scale bar: $20 \mu \mathrm{m} . n=3$.
By taking into account all of these previous observations, we here hypothesize that MG can be reprogrammed in vivo via cell fusion and become a source of new photoreceptors. In the present study, we demonstrate that Wnt-activated HSPCs transplanted in the retina of mouse models of genetic and drug-induced photoreceptor degeneration can fuse with $\mathrm{MG}$ with high efficiency. We found cell fusion to be the functional way through which MG reenters the cell cycle, proliferates, is reprogrammed back to a pluripotent/neural progenitor-like state, and finally differentiates into photoreceptors. Electrophysiological rescue and retinal regeneration are observed, which suggest that MG reprogramming and the subsequent differentiation into photoreceptors via cell fusion is a possible strategy for treatment of retinitis pigmentosa.

\section{Results}

MG cells fuse with transplanted HSPCs upon photoreceptor degeneration. We aimed to determine whether transplanted HSPCs can fuse with MG in a drug-induced mouse model of photoreceptor degeneration. We used Gfap-Cre recipient mice (expressing Cre recombinase under the long form of the Gfap promoter), which restrict expression of Cre to MG $(19,29)$. Indeed, by using the lineage-tracing model that expresses yellow fluorescent protein (YFP) to track MG, we confirmed the exclusive colocalization of YFP with the MG marker glutamine synthase (GS) and not with PKC- $\alpha$ (a marker of bipolar cells), recoverin (a marker of photoreceptors), calbindin (a marker of horizontal cells), calretinin (a marker of amacrine and GCs), or neurofilament, heavy polypeptide (NEFH) and $\beta$-tubulin III (markers of GCs) (Supplemental Figure 1A; supplemental material available online with this article; doi:10.1172/ JCI85193DS1). We treated Gfap-Cre recipient mice with $N$-methyl$\mathrm{N}$-nitroso urea (MNU), which induces progressive photoreceptor cell death (TUNEL ${ }^{+}$) within 24 hours (Figure 1, A and B, and Supplemental Figure 1, B and C; compare WT with MNU/PBS) and complete degeneration of the outer nuclear layer (ONL), where 
A

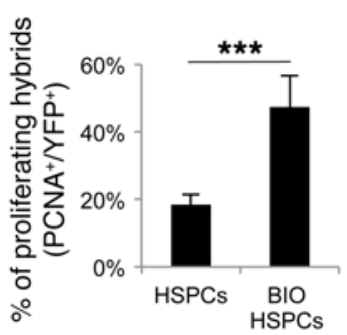

B

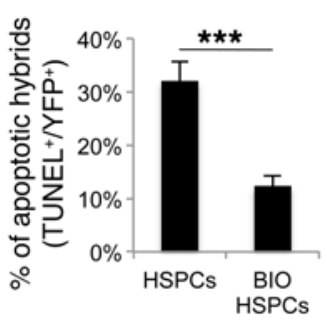

C

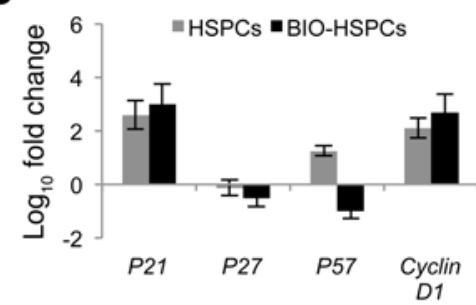

D

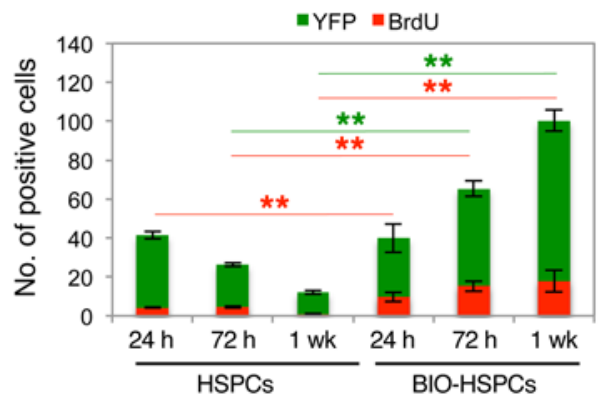

E

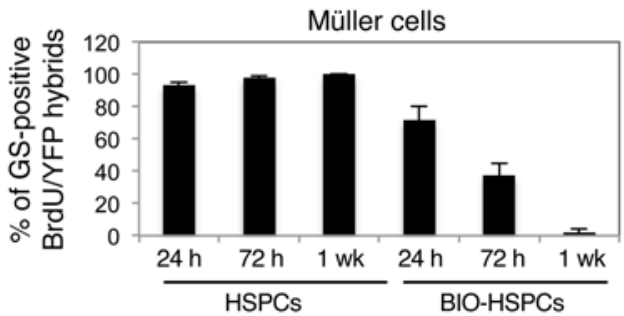

$\mathbf{F}$

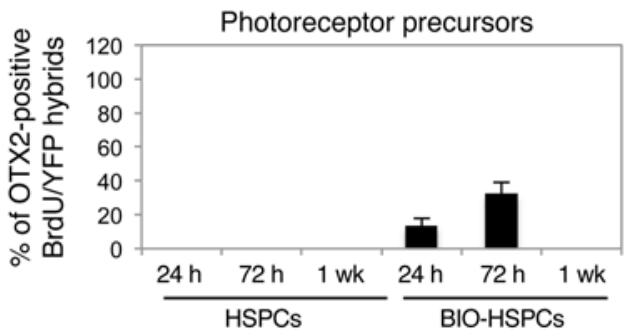

G

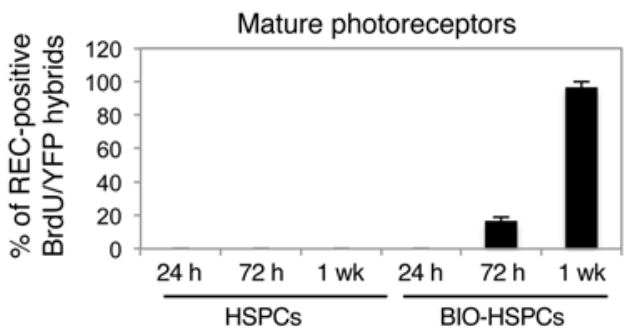

H
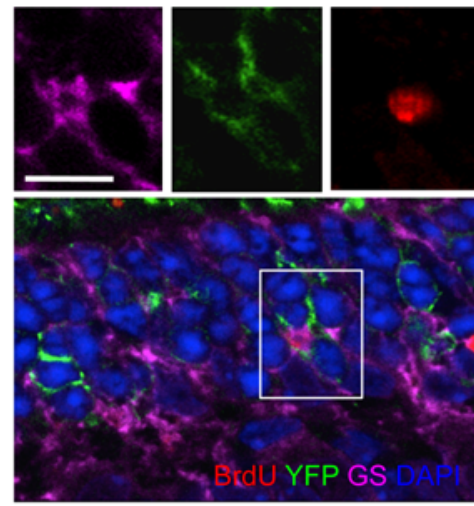

I
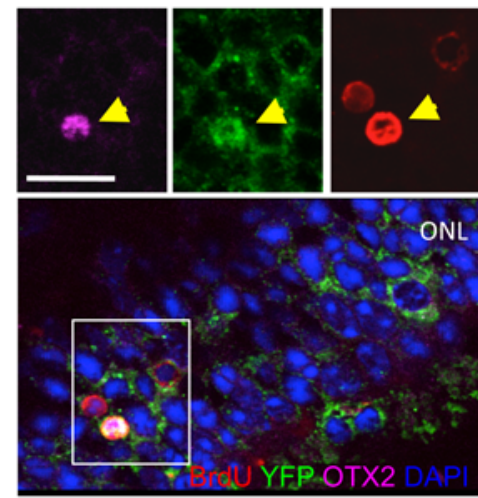

J
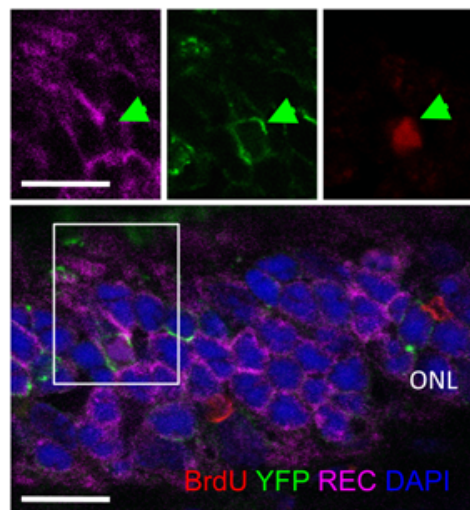

Figure 2. Activation of Wnt signaling promotes proliferation and survival of hybrids. ( $\mathbf{A}$ and $\mathbf{B})$ Percentages of proliferating $\left(\mathrm{PCNA}^{+}, \mathbf{A}\right)$ or apoptotic (TUNEL ${ }^{+}$, B) YFP' ${ }^{+}$hybrids on the total amount of $\mathrm{YFP}^{+}$hybrids in MNU-damaged $\mathrm{Cfap}$-Cre retinas 24 hours after transplantation of untreated (HSPCs) or BIO-treated (BIO-HSPCs) HSPCS ${ }^{\text {R26Y }}$. ${ }^{* *} P<0.0001$, unpaired Student's $t$ test. (C) qPCR analysis of cell-cycle genes on FACS-sorted DiD+YFP+ hybrids harvested 24 hours after transplantation of untreated or BIO-treated HSPCS ${ }^{\text {R26Y }}$ in MNU-damaged $G$ fap-Cre retinas. Data are represented as mean $\pm S D$ of $\log _{10}$ fold changes of gene expression in $\mathrm{DiD}^{+}$ $\mathrm{YFP}^{+}$hybrids with respect to $\mathrm{DiD}^{+} \mathrm{YFP}^{+}$ population-depleted retinas. $n=3$. (D) Total YFP+ hybrids (green bars) that also incorporated BrdU (red bars) detected in MNU-damaged Cfap-Cre retinas 24 hours, 72 hours, and 1 week after transplantation of untreated or BIO-treated HSPCs ${ }^{\mathrm{R} 26 \mathrm{~V}} .{ }^{* *} P<0.001$, 2-way ANOVA and Bonferroni's posttest. Red and green lines show the statistical significance of green (YFP) or red (BrdU) bars. (E-G) Percentages of $\mathrm{YFP}^{+} \mathrm{BrdU}^{+}$hybrids also positive for GS (E, Müller cells), OTX2 (F, photoreceptor progenitors), or recoverin (G, REC, mature photoreceptors) detected in MNU-damaged Gfap-Cre retinas 24 hours, 72 hours, and 1 week after transplantation of untreated or BIO-treated HSPCs ${ }^{\mathrm{R} 26 \mathrm{Y}}$. (H-J) Representative immunostainings of double $\mathrm{YFP}^{+}$(green)/BrdU+ (red) hybrids also positive for either GS (magenta in $\mathbf{H}$, white arrowheads), OTX2 (magenta in I, yellow arrowheads), or recoverin (magenta in J, green arrowheads) stainings in MNU-damaged Cfap-Cre retinas 24 hours (H), 72 hours (I), and 1 week (J) after transplantation of BIO-treated HSPCs. Nuclei were counterstained with DAPI (blue). Images on the top show higher magnification and single channels of areas in the white squares. Scale bars: $20 \mu \mathrm{m}$. (A, B, and D-C) Values in graphs are represented as mean $\pm \operatorname{SD}(n=9)$. photoreceptors are localized, after 7 days (30, 31). Twenty-four hours after MNU damage, we injected HSPCs isolated from LoxPSTOP-LoxP-YFP (R26Y) transgenic donor mice into the subretinal space of Gfap-Cre recipient eyes, close to the layer of damaged photoreceptors. Gfap-Cre mice not treated with MNU were also transplanted as controls. Twelve hours after $\mathrm{HSPCs}^{\mathrm{R} 26 \mathrm{Y}}$ transplantation, we detected many $\mathrm{YFP}^{+}$cells close to the site of the injection; this was due to the stop codon excision by Cre after fusion of HSPCs ${ }^{\mathrm{R} 26 \mathrm{Y}}$ with Müller cells from Gfap-Cre mice $\left(\mathrm{MG}^{\mathrm{Gfap}-\mathrm{Cre}}\right)$ (Figure 1, A and B, and Supplemental Figure 1D). The $\mathrm{YFP}^{+}$hybrids localized radially across the thickness of the retina, which is typical of MG morphology. $\mathrm{YFP}^{+}$hybrids were detected upon transplantation in MNU-damaged retinas, where many photoreceptors were apoptotic (Figure 1B, TUNEL ${ }^{+}$), but they were not appreciable in sections where $\mathrm{HSPCs}^{\mathrm{R} 26 \mathrm{Y}}$ were transplanted into nondamaged Gfap-Cre eyes, which suggests that photoreceptor cell death is necessary to induce cell fusion (Figure 1B). A mean of $5.9 \% \pm 1.5 \%$ of the total transplanted DiD-labeled HSPCs ${ }^{\mathrm{R} 26 \mathrm{Y}}$ was also $\mathrm{YFP}^{+}$when inject- 
A

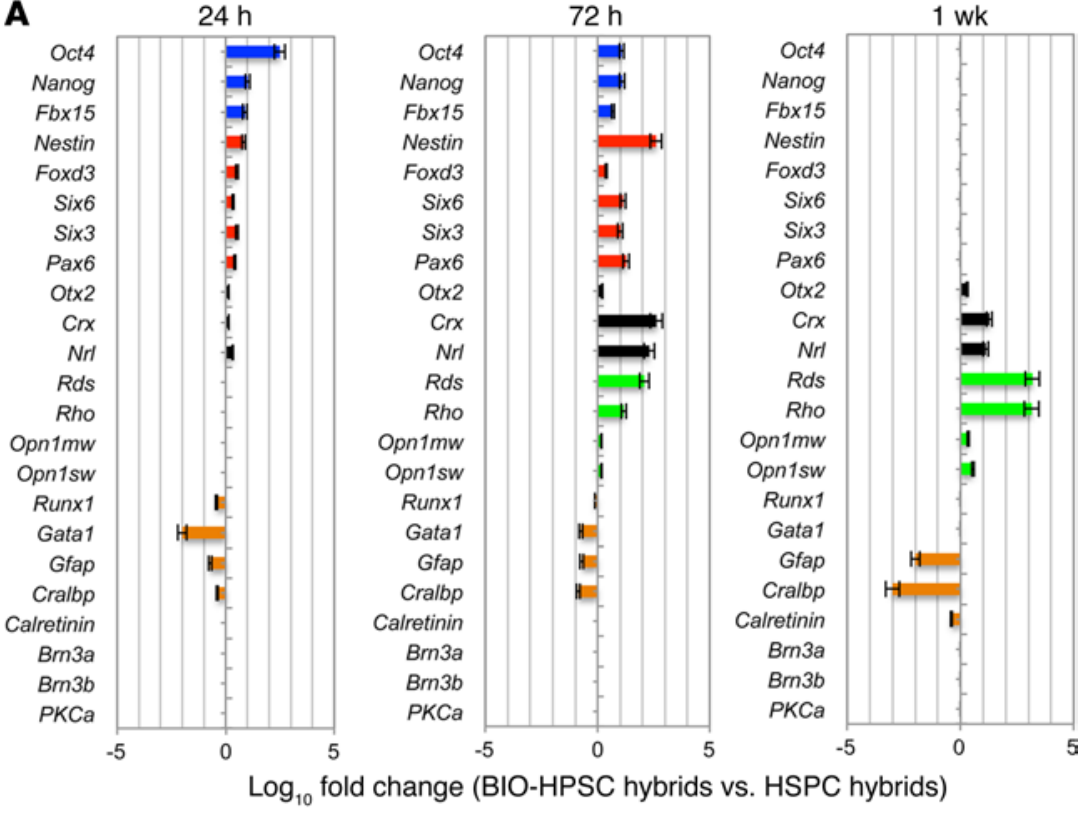

B

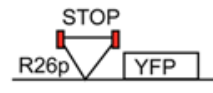

Donor HSPCs ${ }^{\text {R26Y }}$

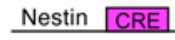

Recipient mouse
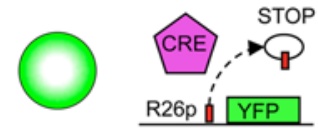

Reprogrammed hybrids
C

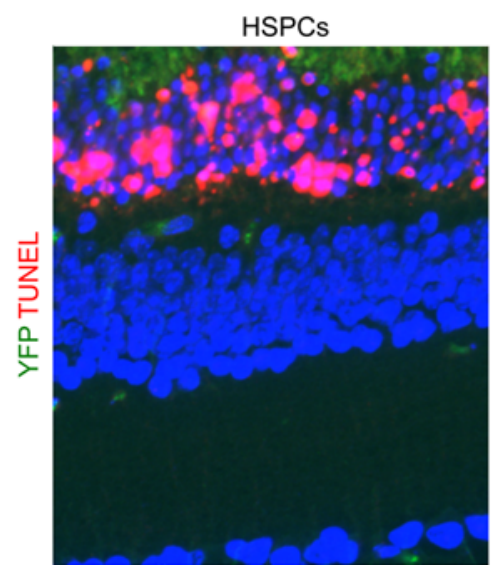

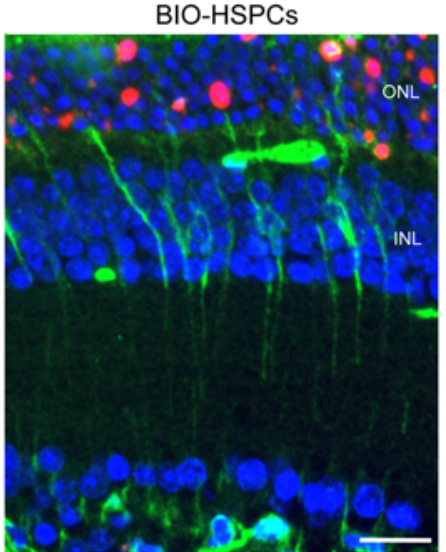

ed into MNU-damaged eyes $\left(\mathrm{DiD}^{+} \mathrm{YFP}^{+}\right.$hybrids; Figure $1 \mathrm{C}$ and Supplemental Figure 1, E and H; Gfap-Cre), which corresponds to the frequency of cell-fusion events. However, a few YFP'DiD hybrids (not included in the analysis) were also found; thus, we cannot exclude an underestimation of fusion frequency. In contrast, we observed very few hybrids (detectable only by FACS analysis and likely due to the mechanical damage induced by the injection) when DiD-labeled $\mathrm{HSPCs}^{\mathrm{R} 26 \mathrm{Y}}$ was transplanted into nondamaged (control) Gfap-Cre recipient eyes (Figure 1C and Supplemental Figure 1H; Gfap-Cre, see below), thus confirming that cell fusion between HSPCs and MG can occur in the presence of tissue damage.

Cell fusion was also confirmed by transplanting DiD-labeled HSPCs expressing Cre under the BM-specific promoter Vav (referred to here as HSPCs ${ }^{\text {Vav-Cre }}$ ) into MNU-damaged and nondamaged R26Y
Figure 3. Activation of Wnt signaling promotes reprogramming of hybrids. (A) Gene expression analysis of pluripotent (blue bars), neural progenitor (red bars), photoreceptor progenitor (black bars), mature photoreceptor (green bars), and differentiated cell (orange bars) markers in $\mathrm{DiD}^{+} \mathrm{YFP}^{+}$hybrids. The hybrids were FACS sorted 24 hours, 72 hours, and 1 week after transplantation of untreated or BIO-treated

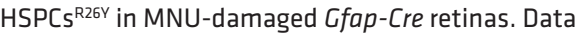
represent the mean of $\log _{10}$ fold changes \pm SEM of gene expression detected in hybrids obtained upon fusion with $\mathrm{BIO}$-treated DiD-HSPCs with respect to hybrids obtained upon fusion with untreated DiD-HSPCs. $n=3$. (B) Schematic representation of the experimental plan to detect dedifferentiation of retinal cells upon fusion. Reactivation of the nestin promoter in retinal cells fused with transplanted HSPCs ${ }^{\mathrm{R} 26 \mathrm{Y}}$ leads to Cre expression and the consequent formation of $\mathrm{YFP}^{+}$hybrids. (C) Representative immunostaining of $\mathrm{YFP}^{+}$hybrids (green) and TUNEL ${ }^{+}$apoptotic photoreceptors (red) on retinal sections obtained from MNU-damaged nestin-Cre retinas 24 hours after transplantation of untreated or BIO-treated $\mathrm{HSPCS}^{\mathrm{R} 26 \mathrm{~V}}$. Nuclei were counterstained with DAPI (blue). Scale bar: $20 \mu \mathrm{m}$.

eyes, where a mean of $7.5 \% \pm 1.2 \% \mathrm{DiD}^{+} \mathrm{YFP}^{+}$ hybrids was found 24 hours after transplantation, but only in the damaged group (Supplemental Figure 1, F-H; HSPCs/R26Y).

Given that we obtained approximately the same percentages of hybrids after transplantation into R26Y or into Gfap-Cre recipient mice (Supplemental Figure $1 \mathrm{H}$ ), we hypothesized that HSPC $^{\text {Vav-Cre }}$ fused mainly with MG upon photoreceptor damage. Furthermore, $\mathrm{YFP}^{+}$hybrids were immunoreactive to the MG marker GS, but not to the photoreceptor marker recoverin (Figure 1D), which further suggests fusion of HSPCs with MG. Finally, almost no $\mathrm{YFP}^{+}$hybrids were identified in both damaged and undamaged retinas harvested 24 hours after transplantation of DiD-labeled HPSCs from R26Y mice $\left(\mathrm{HSPCs}^{\mathrm{R} 26 \mathrm{Y}}\right.$ ) either in recipient mice carrying Cre under the photoreceptor-specific rhodopsin promoter (Rho-Cre; ref. 32) or in recipient mice carrying Cre under the ganglion- and amacrine-specific calretinin promoter (Calr-Cre; ref. 33), thus excluding major fusion of transplanted HSPCs with other retinal cell types (Supplemental Figure 1, H-J). Taken together, these results indicate that HSPCs can fuse almost exclusively with MG when transplanted in the subretinal space of an MNU-induced mouse model of photoreceptor degeneration.

$M G$ reenters the cell cycle upon fusion with HSPCs through a Wnt-dependent process. MG can reenter the cell cycle after retinal damage (14). Furthermore, we previously demonstrated that Wnt signaling enhances the proliferation of hybrids formed in vitro and in vivo $(20,28)$. Thus, we investigated whether MG-derived hybrids can reenter the cell cycle upon Wnt activation. We transplanted into the eyes of MNU-damaged Gfap-Cre mice either $\mathrm{HSPCs}^{\mathrm{R} 26 \mathrm{Y}}$, where the Wnt signaling pathway was preactivated by the GSK-3 inhibitor 6-bromoindirubin-3'-oxime (BIO) (Sup- 
A
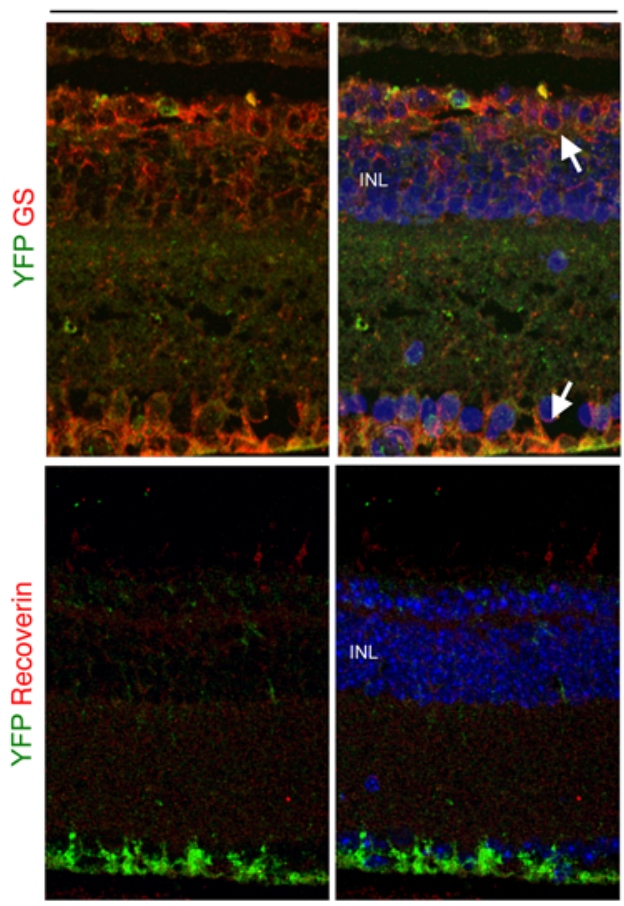

C

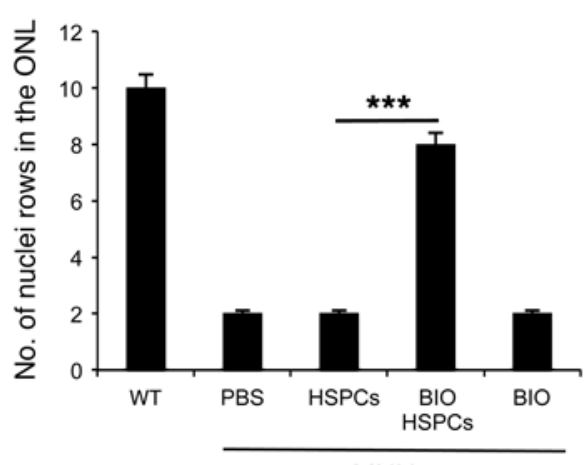

MNU
B
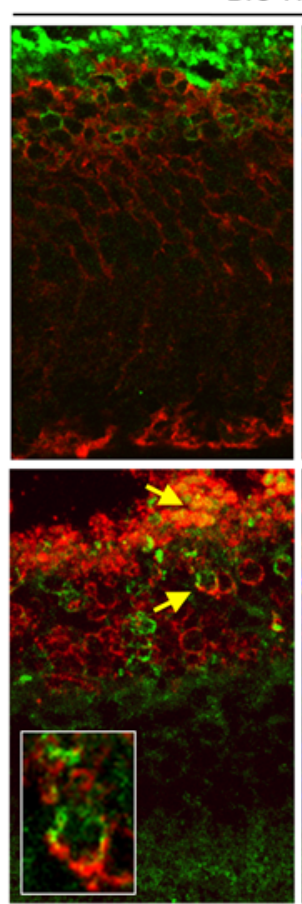

D

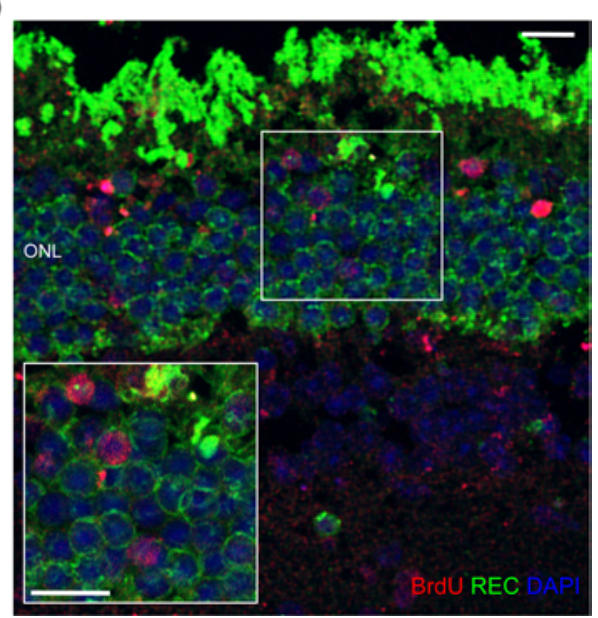

Figure 4. MG reprogrammed upon fusion with Wnt-activated HSPCs generates new photoreceptors. (A and B) Representative immunostainings of $\mathrm{YFP}^{+}$(green) hybrids also immunoreactive for either CS (red, white arrows) or recoverin (red, yellow arrows) detected in MNU-damaged Gfap-Cre retinas 1 week after transplantation of untreated (A) or BIO-treated (B) HSPCS ${ }^{\text {R26Y }}$. Nuclei were counterstained with DAPI (blue). $n=3$.

(C) Statistical analysis of the number of photoreceptor nuclear rows in the ONL of healthy (WT) or MNU-damaged eyes 1 week after treatment with either PBS or untreated (HSPCs) or BIO-treated HSPCs (BIO-HSPCs) or BIO alone. Data are represented as mean $\pm S D$ counted in 3 different sections spanning the site of the injection for each mouse. $n=9$. ${ }^{* * *} P<0.0001$, unpaired Student's $t$ test. (D) Representative immunodetection of BrdU-positive cells (red) also positive for the photoreceptor marker recoverin (green) in the ONL of MNU-damaged Gfap-Cre retinas 1 week after transplantation of BIO-treated $\mathrm{HSPCS}^{\mathrm{R} 26 \mathrm{Y}}$. Nuclei were counterstained with DAPI (blue). Insert represents a higher magnification of the region in the white square. $(n=3)$. Scale bars: $20 \mu \mathrm{m}$. plemental Figure 2A), or nonactivated $\mathrm{HSPCs}^{\mathrm{R} 26 \mathrm{Y}}$. We found $\mathrm{YFP}^{+}$ hybrids that were also positive for the proliferation marker proliferating cell nuclear antigen (PCNA) in sections of retinas harvested 24 hours after transplantation of $\mathrm{HSPCs}^{\mathrm{R} 26 \mathrm{Y}}$ (Figure 2A and Supplemental Figure 2B, HSPCs). However, the majority of these embarked upon apoptosis, as more than $35 \%$ of $\mathrm{YFP}^{+}$cells were also positive for TUNEL staining (Figure $2 \mathrm{~B}$ and Supplemental Figure 2, C and D, HSPCs). In contrast, more $\mathrm{PCNA}^{+}$hybrids were detected 24 hours after transplantation of BIO-treated HSPCs (Figure 2A and Supplemental Figure 2B, BIO-HSPCs) and only a few $\mathrm{YFP}^{+}$hybrids underwent apoptosis (Figure 2B and Supplemental Figure 2, C and D, BIO-HSPCs). These results suggest that preactivation of Wnt signaling in HSPCs enhances the survival and proliferation of hybrids formed upon fusion with MG. Of note, Wnt activation did not increase the fusion efficiency (Supplemental Figure 2E; compare HSPCs with BIO-HSPCs).
We then investigated the expression levels of different cell-cycle regulators in hybrids that were FACS sorted 24 hours after transplantation of BIO-treated or untreated $\mathrm{HSPCs}^{\mathrm{R} 26 \mathrm{Y}}$ in MNU-damaged Gfap-Cre eyes. Cyclin D1, which is expressed in mitotic retinal progenitor cells and is required for progenitor cell proliferation $(34,35)$, was strongly upregulated in hybrids formed after transplantation of both BIO-treated and untreated HSPCs (Figure 2C). We also found upregulation of $\mathrm{p} 21^{\text {(Cip1/WAF1), }}$ which is correlated with neural stem cell maintenance and tissue regeneration (36). Interestingly, the cyclin kinase inhibitors $\mathrm{p} 27^{\mathrm{kip} 1}$, which is downregulated in activated MG (37), and p57 $7^{\mathrm{kip} 2}$, which is associated with cell-cycle exit of retinal progenitor cells (14), were both downregulated in hybrids obtained after transplantation of BIO-treated HSPCs (Figure 2C), which suggests the prolonged proliferative potential of the BIO hybrids. These observations indicate that some cell-cycle genes are 
perturbed in both types of hybrids; however, the activation of HSPCs with BIO provides a proliferative advantage.

To further analyze the fate of the proliferating hybrids, we injected BrdU twice every 12 hours in MNU-damaged Gfap-Cre mice that were transplanted with untreated or BIO-treated $\mathrm{HSPCs}^{\mathrm{R} 26 \mathrm{Y}}$. Thus, we analyzed $\mathrm{YFP}^{+}$hybrids that incorporated BrdU 24 hours, 72 hours, and 1 week after transplantation. Although the total number of $\mathrm{YFP}^{+}$hybrids detected in each retinal section was similar 24 hours after transplantation of either untreated or BIO-treated HPSCs, Wnt signaling activation resulted in more hybrids that incorporated BrdU (Figure 2D), which indicates their proliferative advantage. Interestingly, 72 hours and 1 week after transplantation of untreated HSPCs, the total number of $\mathrm{YFP}^{+}$hybrids was reduced, which suggests that hybrids that did not proliferate (i.e., $\mathrm{YFP}^{+} \mathrm{BrdU}^{-}$cells) possibly died (Figure 2D). In contrast, the number of double-positive $\mathrm{BrdU}^{+} \mathrm{YFP}^{+}$ cells as well as the number of total $\mathrm{YFP}^{+}$hybrids increased 72 hours and 1 week after transplantation of BIO-treated HSPCs, which suggests that upon activation of Wnt signaling, some proliferating $\mathrm{BrdU}^{+} \mathrm{YFP}^{+}$hybrids had moved into and through a new $\mathrm{M}$ phase and survived. Moreover, we also detected a higher number of $\mathrm{YFP}^{+} \mathrm{BrdU}^{-}$ cells 72 hours and 1 week after transplantation of BIO-HSPCs, with respect to those counted 24 hours after transplantation (Figure 2D), which suggests that either some proliferating hybrids did not incorporate BrdU or that they underwent multiple divisions and thus lost the BrdU. Together, these results suggest that activation of Wnt signaling in transplanted HSPCs promotes proliferation and survival of hybrids formed after fusion with MG.

$M G$ is reprogrammed in vivo after fusion with Wnt-activated HSPCs, and the hybrids differentiate into photoreceptors. We aimed to determine whether MG is reprogrammed in vivo upon fusion with Wnt-activated HSPCs. Interestingly, we observed that, while the majority of the proliferating $\mathrm{YFP}^{+} \mathrm{BrdU}^{+}$hybrids were positive for the MG marker GS 24 hours, 72 hours, and 1 week after transplantation of untreated HSPCs, the percentage of $\mathrm{YFP}^{+} \mathrm{BrdU}^{+}$ hybrids positive for GS was drastically reduced 72 hours and 1 week after transplantation of BIO-HSPCs (Figure 2, E and H, see below) and the latter were mainly positive for the expression of the photoreceptor progenitor marker orthodenticle homeobox 2 (OTX2) at 72 hours after transplantation (Figure 2, F and I, see below). Furthermore, we noted that the photoreceptor marker recoverin started to be expressed in the $\mathrm{YFP}^{+} \mathrm{BrdU}^{+}$hybrids 72 hours after transplantation of BIO-HSPCs and that all the $\mathrm{Brdu}^{+}$ $\mathrm{YFP}^{+}$cells expressed recoverin 1 week after transplantation (Figure 2, G and J, see below). Of note, the proliferating hybrids (i.e., double $\mathrm{YFP}^{+} \mathrm{BrdU}^{+}$hybrids) were mainly localized in the ONL already at 24 hours (Figure $2 \mathrm{H}$ ), when they expressed MG markers, and consequently, at late time points (Figure 2J, see below), when they differentiated toward photoreceptors, which indicates their movement toward the ONL. These results suggest that Wnt signaling promotes the reprogramming of the proliferating hybrids toward a photoreceptor progenitor fate.

To further strengthen these results, $\mathrm{DiD}^{+} \mathrm{YFP}^{+}$hybrids were FACS sorted 24 hours, 72 hours, and 1 week after transplantation of DiDlabeled BIO-HSPCs ${ }^{\mathrm{R} 26 \mathrm{Y}}$ or untreated DiD-HSPCs ${ }^{\mathrm{R} 26 \mathrm{Y}}$ in MNU-damaged Gfap-Cre eyes. The gene expression profiles were analyzed (Figure 3A). Twenty-four hours after transplantation, pluripotent markers (Oct4, Nanog, and Fbx15) were upregulated in the hybrids obtained after fusion of BIO-HSPCs and progressively silenced 1 week after transplantation. The Wnt target Axin2 was also upregulated in the hybrids 24 hours after transplantation of BIO-HSPCs, which indicates activation of Wnt signaling (Supplemental Figure 3A). In contrast, neural progenitor markers (Nestin, Foxd3, Six6, Six3, and Pax6) were upregulated up to 72 hours after transplantation, while they were silenced in 1 week. The postmitotic photoreceptor progenitors (Otx2, $C r x$, and $N r l$ ) were upregulated up to 1 week after transplantation. Finally, the mature rod photoreceptor markers peripherin $(R d s)$ and rhodopsin (Rho) were upregulated already 72 hours after transplantation, but were very highly expressed within 1 week. Low upregulation of the cone photoreceptor markers $O p n 1 m w$ and $O p n 1 s w$ was also detected. However, no upregulation of calretinin (Calr, expressed in ganglion and amacrine cells) nor of Brn3a, Brn3b (markers of GCs) nor of $P k c a$ (a marker of bipolar cells), was detected at any time point, which suggests that hybrids were not differentiated toward these retinal cell fates. The HSPC (Runx1, Gata1) and MG (Gfap, Cralbp) markers were downregulated already 24 hours after transplantation (Figure 3A). For the hybrids obtained after the fusion of untreated HSPCs, we did not observe upregulation of the above-mentioned markers; instead, HSPC (Runx1, Gata1) and MG (Gfap, Cralbp) genes were expressed (Supplemental Figure 3B). These data indicated that the hybrids formed after fusion of BIO-HSPCs underwent reprogramming to a pluripotent/neural progenitor-like fate and then committed toward photoreceptors in vivo.

To confirm this conclusion, we transplanted BIO-treated or untreated $\mathrm{HSPCs}^{\mathrm{R} 26 \mathrm{Y}}$ in the eyes of MNU-damaged nestin-Cre recipient mice (Figure 3B). We observed $\mathrm{YFP}^{+}$hybrids only after transplantation of BIO-HSPCs (Figure 3C), which indicates that reactivation of nestin and reprogramming of the hybrids was dependent on Wnt activity. Furthermore, upon MNU treatment, in 24 hours, photoreceptors start to die $\left(\mathrm{TUNEL}^{+}\right)$in retinas transplanted with either untreated HSPCs, BIO alone, or vehicle alone (PBS). Instead, a much-reduced number of TUNEL ${ }^{+}$ photoreceptors was seen in BIO-HSPC-transplanted retinas and especially in the proximity of $\mathrm{YFP}^{+}$hybrids (Figure 3C and Supplemental Figure 1, B and C).

We then verified on sections whether Müller-derived hybrids can generate photoreceptors. We performed double staining of retinal sections 1 week after transplantation of BIO-treated or untreated HSPCs in MNU-damaged Gfap-Cre eyes (Figure 4, A and B). First, upon HSPC transplantation, we observed only 1 or 2 leftover nuclear layers in the ONL, and these were negative for the expression of recoverin (Figure 4, A and C, and Supplemental Figure $4 \mathrm{~A}$ ). The few $\mathrm{YFP}^{+}$hybrids detected were positive for the MG marker GS (Figure 4A). In contrast, after transplantation of BIO-treated HSPCs, we found up to 8 nuclear layers in the ONL (Figure 4, B and C, and Supplemental Figure 4A), which included hybrids positive for both YFP and recoverin (i.e., photoreceptors) (Figure 4B).

Overall, these results show that MG can be efficiently reprogrammed into photoreceptors upon fusion with BIO-treated HSPCs, passing through a proliferating and more dedifferentiated intermediate.

Despite many photoreceptors derived from the proliferation and differentiation of reprogrammed MG cells (i.e., BrdU/Rec double-positive cells; Figure 4D), not all of the photoreceptors in 
A
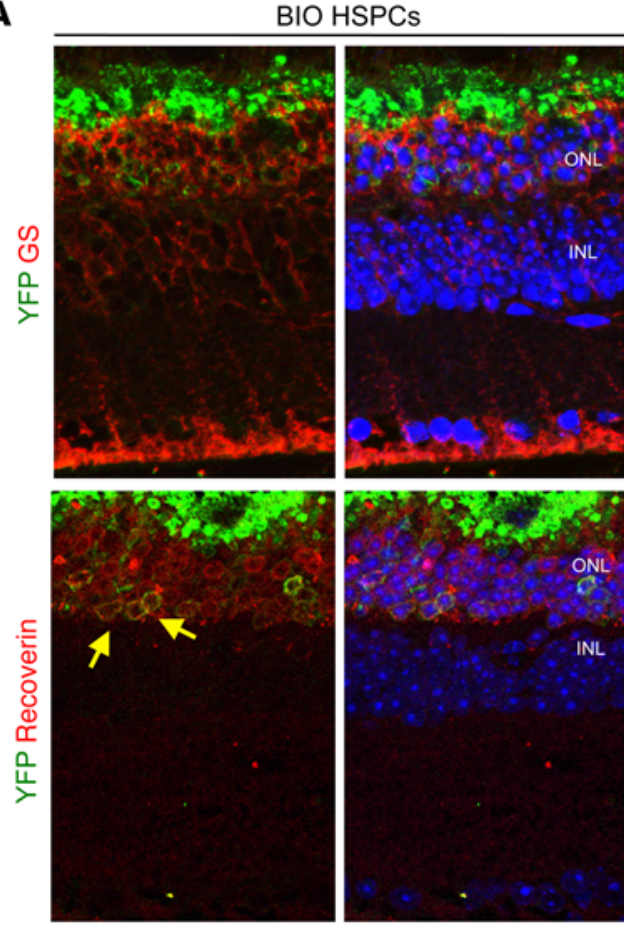

B
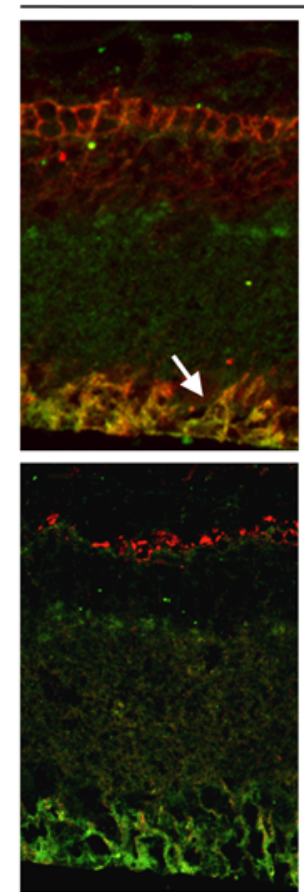

HSPCs
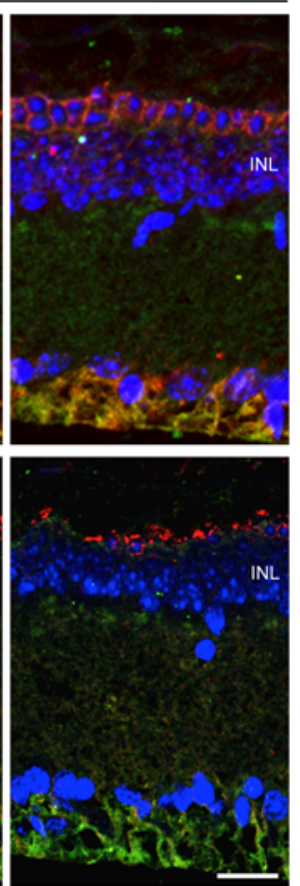

C

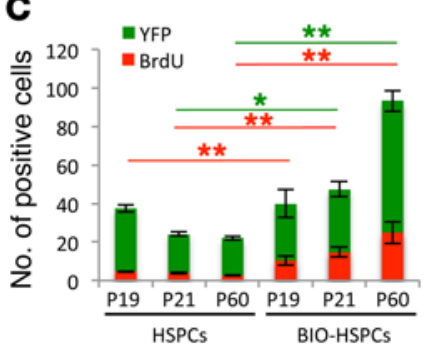

D

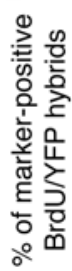

E

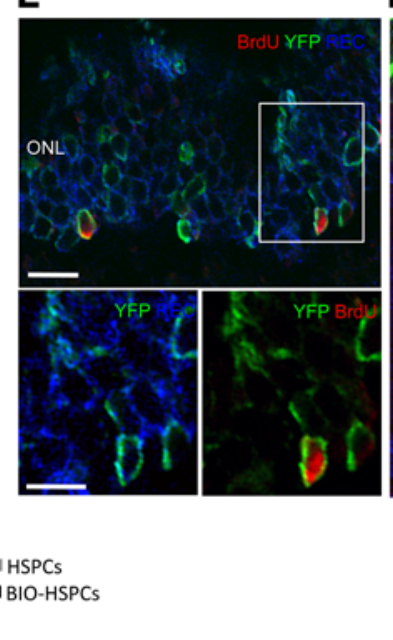

$\mathbf{F}$

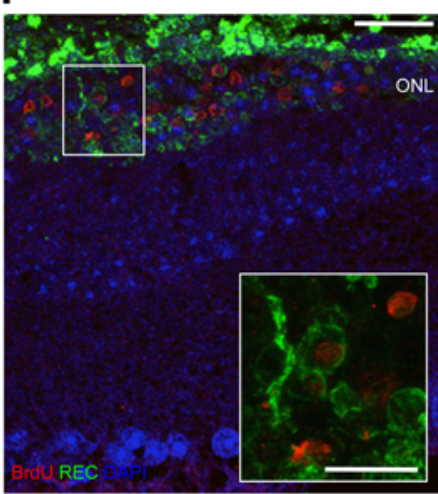

Figure 5. Regeneration of photoreceptors in rd10 mice. (A and B) Representative immunostainings of $\mathrm{YFP}^{+}$(green) with either CS (red, white arrow) or recoverin (red, yellow arrows) in $r d 10^{\mathrm{R} 26 \mathrm{~V}}$ retinas 42 days after transplantation of BIO-treated (A) or untreated (B) HSPCs ${ }^{\text {Vav-Cre }}, n=3$. (C) Total number of $\mathrm{YFP}^{+}$hybrids (green bars) that incorporated BrdU (red bars) detected in rd10 $26 \mathrm{Y}$ eyes 24 hours (P19), 72 hours (P21), and 42 days (P60) after transplantation of untreated or BIO-treated $\mathrm{HSPCs}^{\text {Vav-cre }}$.

Data are represented as mean \pm SD. $n=9$. ${ }^{*} P<0.001 ;{ }^{*} P<0.01,2$-way ANOVA and Bonferroni's post-test. Red and green lines show the statistical significance of the difference between the respective green (YFP) and red (BrdU) bars. (D) Percentages of double YFP+BrdU' hybrids also positive for $\mathrm{CS}$, OTX2, or recoverin stainings 42 days (P60) after transplantation of untreated (gray bars) or BIO-treated ( black bars) HSPCs ${ }^{\text {Vav-Cre in }}$ rd10 ${ }^{\text {R26Y }}$ retinas. Data are represented as mean $\pm \mathrm{SD} . n=9$. (E) Representative immunodetection of $\mathrm{BrdU}^{+}$cells (red) also positive for the photoreceptor marker recoverin (green) in the ONL of MNU-damaged $r d 10^{\text {R26V }}$ retinas 42 days after transplantation of BIO-treated HSPCs ${ }^{\text {Vav-Cre. }}$. Nuclei were counterstained with DAPI (blue). Insert shows higher magnification of the region in the white square. (F) Representative confocal image of YFP+ hybrids (green) also positive for $\mathrm{BrdU}$ (red) and recoverin (blue) in the ONL of $r d 10^{\mathrm{R} 26 \mathrm{~V}}$ retinas 42 days after transplantation of BIO-treated HSPCs ${ }^{\text {vav-Cre }}$. Images at the bottom represent enlargements of the same YFP+ hybrid (green) also positive for recoverin (blue in the left panel) and for BrdU (red in the right panel) stainings. $n=3$. Scale bar: $20 \mu \mathrm{m}$.
BIO-HSPC transplanted retinas were derived from the hybrids, as not all of them were positive for YFP (Figure 4B) or for BrdU (Figure $4 \mathrm{D}$ ), which suggests that newly generated photoreceptors can contribute overall to blocking the death of the neighboring ones.

In vivo reprogramming of $M G$ upon fusion with Wnt-activated HSPCs can rescue retinal degeneration phenotypes. We next aimed to investigate the possible functional rescue of the retinal degeneration phenotype due to the photoreceptors derived from the hybrids formed by fusion of MG and BIO-treated HSPCs. Although MNU-induced photoreceptor cell death is a useful model for studying the effects of therapeutic approaches to treating retinal degeneration in the short term, this alkylating drug leads to extensive oxidative stress and induction of tumors within 1 month $(38,39)$. Thus, we determined whether MG-derived photoreceptors can functionally rescue the photoreceptor degeneration phenotype in a type 10 retinal degeneration mouse model $(r d 10)$ over the long term. This is a model of inherited autosomal recessive retinitis pigmentosa, which carries a spontaneous mutation in the rod-specific $\beta$ subunit of cyclic guanosine monophosphate-phosphodiesterase (cGMP-phosphodiesterase) (PDE6B). The rd10 mice show photoreceptor dysfunction starting from P18 (i.e., 18 days after birth), and full rod photoreceptor degeneration is followed by cone degeneration and remodeling of the inner retina by P40 (40).

First, we confirmed the presence of $\mathrm{YFP}^{+} \mathrm{DiD}^{+}$hybrids after transplantation of BIO-treated or untreated DiD-labeled HSPCs ${ }^{\text {Vav-Cre }}$ in the subretinal space of P18 rd10 mice carrying the R26Y transgene (referred to as $r d 10^{\mathrm{R} 26 \mathrm{Y}}$ ) when the degeneration started (Supplemental Figure 5, A and B). $\mathrm{YFP}^{+} \mathrm{DiD}^{+}$hybrids were not detected in transplanted WT control retinas, which indicated that $r d 10$ photoreceptor degeneration induced fusion of MG with transplanted 
A

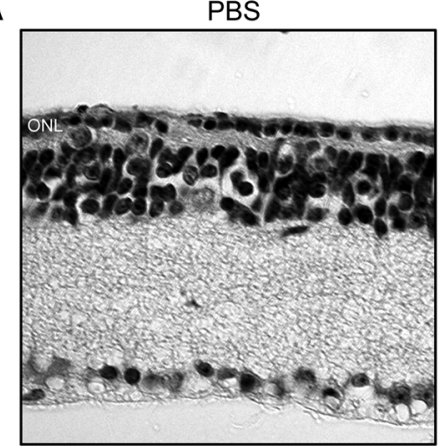

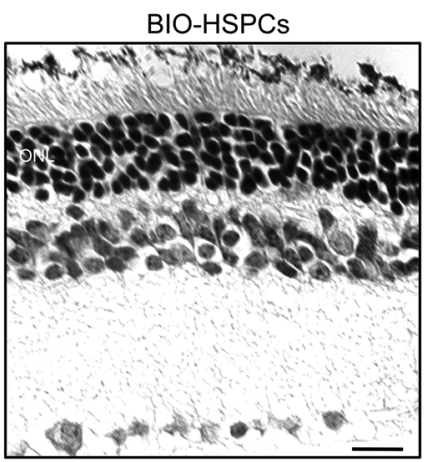

B

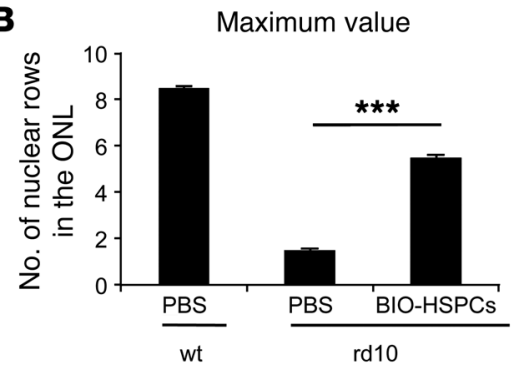

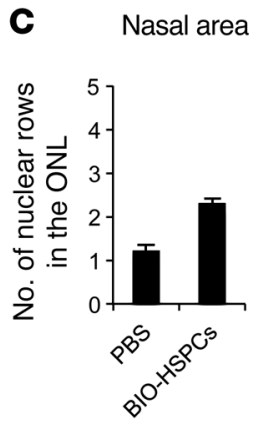
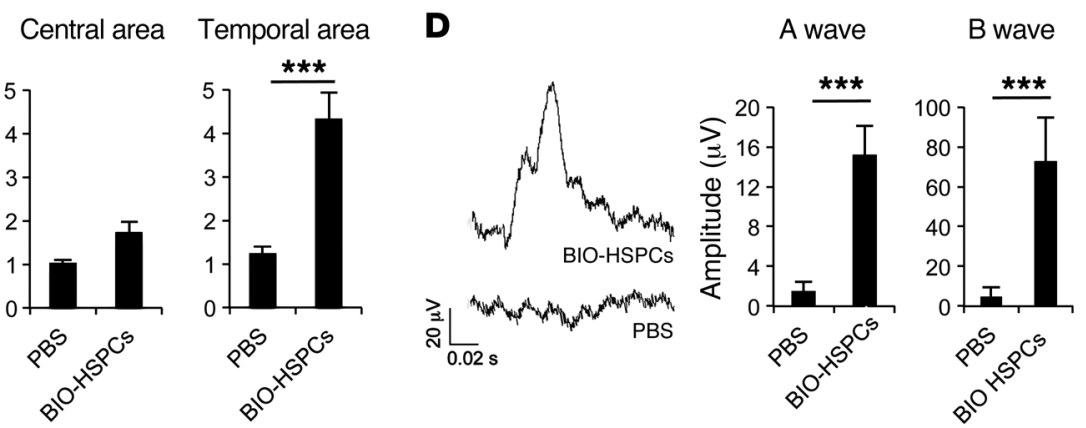

E

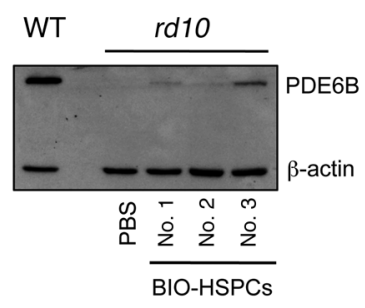

Figure 6. Functional rescue of the rd10 phenotype upon BIO-treated HSPC transplantation. (A) Representative H\&E staining of 2-month-old rd10 retinas

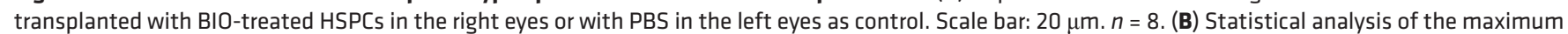
number of nuclear rows detected in the ONL of 2-month-old mouse retinas harvested from either WT or rd10 animals transplanted at P18 with BIO-treated HSPCs or treated with vehicle alone (PBS) as control. Data are represented as mean \pm SEM. $n=8$. ${ }^{* *} P<0.0001$, unpaired Student's $t$ test. (C) Statistical analysis of the number of ONL nuclei rows detected in the nasal, central, or temporal retinal areas of 2-month-old rd10 right retinas transplanted with BIO-treated HSPCs with respect to left control (PBS) eyes. ${ }^{* *} P<0.0001$, unpaired Student's $t$ test. Nine serial sections for each mouse ( $n=8$ ) were analyzed. Counts of 8 retinas out of 21 showed increased nuclear rows and were plotted. (D) Representative ERG responses of 2 -month-old $r d 10$ mice transplanted in the right eyes with BIO-treated HSPCs (BIO-HSPCs) or with vehicle alone (PBS) in left eyes as control. Data are represented as mean \pm SD ( $n=8$ ) of the A-wave and B-wave amplitudes $(\mu \mathrm{V})$ of ERG responses from 8 out of 21 treated mice that showed increased ONL thickness upon BIO-treated HSPC transplantation. ${ }^{* *} P<0.0001$, unpaired Student's $t$ test. (E) Western blotting of PDE6B protein levels in retinas harvested from either 2 -month-old WT or rd10 mice treated with PBS or transplanted with BIO-treated HSPCs at P18. Three different BIO-HSPC transplanted retinas were analyzed (no. 1, no. 2, no. 3).

HSPCs (Supplemental Figure 5, A and B). To confirm fusion with MG, we also transplanted HSPCs ${ }^{\text {Vav-Cre }}$ in $\mathrm{P} 18$ rd10 $^{\mathrm{R} 26 \mathrm{Y}}$; as expected, 24 hours after transplantation, the hybrids were immunoreactive to the MG marker GS, but not to the photoreceptor marker RHO (Supplemental Figure 5, C and D). The hybrids obtained upon fusion with BIO-treated HSPCs ${ }^{\text {Vav-Cre }}$ proliferated (Supplemental Figure 5E) and expressed pluripotent/neural progenitor markers (Supplemental Figure 5G, see below). In contrast, the $\mathrm{YFP}^{+}$hybrids formed upon transplantation of untreated HSPCs ${ }^{\mathrm{Vav}-\mathrm{Cre}}$ underwent apoptosis (Supplemental Figure 5F) and were not reprogrammed (Supplemental Figure 5G).

Next, 42 days after transplantation, at P60, we analyzed the presence of hybrid-derived photoreceptors. Interestingly, in 2-month-old rd10 mice transplanted with BIO-treated HPSCs isolated from Vav-Cre/CAG-RFP double-transgenic animals (where RFP indicates red fluorescent protein; HSPCs ${ }^{\mathrm{Vav}-\mathrm{Cr} / \mathrm{RFP}}$ ), many $\mathrm{YFP}^{+} \mathrm{RFP}^{+}$hybrids were detected in the regions of the ONL, including a high number of nuclear rows (Supplemental Figure $5 \mathrm{H}$ ). Indeed, 42 days (at P60) after transplantation of BIO-treated HSPCs ${ }^{\text {Vav-Cre }}$, we found $\mathrm{YFP}^{+}$hybrids that were positive for recoverin staining and negative to GS (Figure $5 \mathrm{~A}$ ). In contrast, as expected, few $\mathrm{YFP}^{+}$hybrids were found in HSPC-transplanted rd10 retinas of 2-month-old mice. When detectable in sections, these hybrids were positive for the MG marker GS and negative for recoverin (Figure 5B). Moreover, while only 1 row of nuclei in the ONL was detected in $r d 10$ eyes transplanted with untreated HSPCs, a much higher number of photoreceptor nuclei was observed in mice transplanted with BIO-treated HSPCs (Figure 5, $\mathrm{A}$ and $\mathrm{B})$.

We then performed cell-tracing experiments to investigate whether the hybrid-derived photoreceptors were generated from proliferative events. We followed $\mathrm{BrdU}^{+}$hybrids after transplantation of BIO-treated and untreated HSPCs ${ }^{\text {Vav-Cre }}$ in $r d 10^{\mathrm{R} 26 \mathrm{Y}}$. Activation of Wnt signaling in transplanted HSPCs resulted in an increase in the proliferating hybrids at 24 hours (P19), 72 hours (P21), and 42 days (P60) after transplantation with respect to the corresponding times after HSPC transplantation (Figure 5C). In contrast, the $\mathrm{YFP}^{+}$hybrids decreased after HSPC transplantation (Figure 5C), which suggests that they underwent cell death in the latter case. $\mathrm{Brdu}^{+} \mathrm{YFP}^{+}$hybrids expressed GS after HSPC transplantation at 24 hours, 72 hours, and 42 days and therefore remained as MG (Figure 5D and Supplemental Figure 5, I and J). Instead, after BIO-HSPC transplantation, $\mathrm{Brdu}^{+} \mathrm{YFP}^{+}$hybrids progressively silenced GS and expressed OTX2 and recoverin, which suggests their differentiation toward a photoreceptor cell fate (Figure 5D and Supplemental Figure 5, I and J). Remarkably, 
by tracing them, we found a large increase of hybrids positive for BrdU in $\mathrm{P} 60$ mice, which indicates that they efficiently proliferated and differentiated toward a mature photoreceptor cell fate (Figure 5, C, E, and F). Of note, not all of the photoreceptors were $\mathrm{BrdU}^{+}$or $\mathrm{YFP}^{+}$, which suggests that in the $r d 10$ model, as in the MNU model, a regeneration process goes along with some preservation effect.

We then counted the number of nuclear photoreceptor rows across sections. Histological analysis of retinal sections from the control left eyes of 2-month-old rd10 mice treated with PBS confirmed complete photoreceptor degeneration, with only 1 row of photoreceptor nuclei detectable in the ONL (Figure 6, A-C). Instead, up to 5 rows of photoreceptor nuclei were detected in the ONL of the right eyes of a group of mice transplanted with BIO-treated HSPCs, specifically in the temporal area where the cells were transplanted (Figure 6, A-C). Of note, no changes were detected in either the nuclear row numbers in the inner nuclear layer (INL) or in the number of GC nuclei, which suggests that treatment with BIO-HSPCs results specifically in the rescue of photoreceptor cell loss (Supplemental Figure 6, A-C).

To investigate whether functional rescue of the $r d 10$ phenotype was achieved, we recorded scotopic electroretinographic (ERG) responses of $r d 10$ dark-adapted right eyes transplanted with BIO-treated HSPCs. Left eyes treated with PBS were also recorded as controls. Remarkably, we observed that some of the retinas transplanted with $\mathrm{BIO}$-treated HSPCs showed recordable A-waves and B-waves (Figure 6D), albeit these were lower than the WT levels (WT A-wave mean $[\mu \mathrm{V}]=416 \pm 77 \mathrm{SD}$; WT $\mathrm{B}$-wave mean $[\mu \mathrm{V}]=826 \pm 107 \mathrm{SD} ; n=4)$. The A-wave is generated from functional rod photoreceptors, while the B-wave is mainly derived from retinal cells making synapses with photoreceptors (41). Interestingly, the mice that showed an increase in the number of photoreceptor nuclear rows (Supplemental Figure 6D) also showed recordable A-waves and B-waves (Supplemental Figure 6E). Instead, the contralateral untransplanted eyes showed completely flat ERG responses (Figure 6D and Supplemental Figure 6E).

Similar results were obtained with the $r d 10^{\mathrm{R} 26 \mathrm{Y}}$ mouse strain, where the retinas of mice displaying $\mathrm{YFP}^{+}$hybrids also showed the highest number of rows and were positive for the ERG (Supplemental Figure 6, $\mathrm{F}$ and $\mathrm{G}$ ).

Subretinal injection of P18 pups is a challenging chirurgical approach, and we therefore observed retinal regeneration in a subset of BIO-HSPC transplanted mice. Therefore, we monitored the outcome of the injection by optical coherence tomography (OCT) in a different group of $r d 10$-transplanted mice. We confirmed that only the group of eyes successfully transplanted with BIO-treated HSPCs showed photoreceptor regeneration at the histological level (Supplemental Figure 6, A-C). In all these mice, the OCT generated a cataract, and it was not possible to record the ERG.

The ERG A-wave is normally flat in 2-month-old $\mathrm{r} d 10$ mice because of the absence of PDE6B, which is necessary for the closure of cGMP-gated cationic rod channels (42). Therefore, we analyzed the levels of the PDE6B protein in retinas harvested from 2-month-old $r d 10$ mice transplanted with untreated or BIO-treated HSPCs at P18. WT retinas treated with PBS were also analyzed as controls. The PDE6B protein was completely absent in untreat- ed $r d 10$ eyes, while it was present at variable levels in 3 different rd10 eyes transplanted with BIO-treated HSPCs (Figure 6E). This suggests that WT PDE6B was expressed by the genome of the transplanted HSPCs within the hybrids, thereby rescuing the $r d 10$ phenotype.

In conclusion, our results demonstrate that, upon fusion with Wnt-activated HSPCs, MG can be efficiently reprogrammed and differentiated into functional photoreceptors in vivo and that this might represent a therapeutic strategy for rescuing retinal degeneration phenotypes.

\section{Discussion}

Retinitis pigmentosa is a family of inherited diseases that cause retinal degeneration due to photoreceptor cell death. The $r d 10$ mouse model reproduces one of the earliest onset and most aggressive forms of recessive retinitis pigmentosa, which is due to mutations in the Pde6b gene $(43,44)$. Very limited therapeutic approaches are currently available for the treatment of this blindness. Indeed, many efforts have been made to block or delay degeneration of photoreceptors $(45,46)$; however, to date, very limited success has been obtained in terms of their regeneration in mouse models of retinitis pigmentosa.

Here, we have shown that MG can be reprogrammed in vivo and, in turn, efficiently differentiated into photoreceptors via cell fusion with HSPCs in a drug-induced model of retinal degeneration and in $r d 10$ mice, provided that Wnt signaling was previously activated in the transplanted cells. BrdU ${ }^{+}$photoreceptors originated from the MG-derived hybrids were tracked over the long term, i.e., 42 days after transplantation of BIO-HSPCs in $r d 10$ mice. On the other hand, we also clearly observed a protective effect of the resident photoreceptors upon transplantation of BIO-HSPCs, which can be due to hybrid-mediated indirect protection or to a direct effect mediated by BIO-HSPC-secreted factors. Therefore, the hybrid-derived photoreceptors likely blocked or delayed the overall retina degeneration; indeed, not all the surviving photoreceptors in the long term after transplantation were YFP positive. Some of them were resident photoreceptors that were probably protected from cell death. Our data also suggest that, while in the degenerated retinas $M G$ appears to extend into the remodeled ONL, upon transplantation of BIO-HSPCs, the ONL is regenerated and MG staining appears in sections as in a WT retina.

We should also consider the possibility that MG phagocytoses BIO-HSPCs and that this could result in either regenerative or protective effect toward the ONL neurons. However, MG cells were shown to phagocytose apoptotic retinal cells during development (47) as well as apoptotic photoreceptors in degenerated mouse retinas $(48,49)$. Here, we observed expression of RFP and Pde $6 b$ long term after transplantation, suggesting that the genome derived from donor transplanted BIO-HSPCs is present and is expressed in the $\mathrm{YFP}^{+}$neurons derived from MG; therefore, phagocytosis is unlikely to occur in our experimental setting. On the other hand, if some BIO-HSPC-transplanted cells are phagocytosed by MG, these will just die and will likely not be the cells that regenerate the photoreceptors.

Wnt signaling controls regeneration in zebrafish and tadpoles $(19,50,51)$. We have previously shown that ganglion and amacrine neurons in the mouse retina can be regenerated by the 
hybrids formed after Wnt-activated HSPCs (28). How is it possible that the hybrids can generate either photoreceptors, GCs, or amacrine neurons? It appears likely that this is dependent on the signaling pathway induced, on the type of damaged cells in need of being replenished, and also on the site of injection. Here, we transplanted HSPCs very close to the photoreceptor layer; we also attempted transplantation in the vitreous, but we did not observe regeneration. Furthermore, transplantation at late times, when the ONL was completely lost, was not effective in rescuing the increase in nuclear rows in the ONL, which indicates that it is essential to transplant mice when some photoreceptors are still present. A fully degenerated photoreceptor layer cannot be reverted, which indicates that the time of intervention is critical. However, in human pathology, where degeneration is much slower than in $r d 10$ mice, it is possible that the time window to transplant the cells will be considerably larger. It is also worth considering that different signals can be activated by damaged neurons and they can induce alternative differentiation responses; for example, MG regenerates photoreceptors when these specific cells are destroyed in goldfish (52). These signals might in principle be targeted to enhancing the efficacy of the therapy.

In vitro-generated neurons after transdifferentiation of somatic cells are an exciting tool for regenerative medicine; however, engraft problems are a bottleneck for this approach. Strategies based on in vivo conversion of resident cells through the overexpression of key transcription factors might overcome this limitation; nevertheless, to reach clinical applications, it is imperative to obtain a safe in vivo delivery system to specifically target cells where the expression of reprogramming factors can be turned off. In this context, cell fusion with endogenous glial cells represents a promising therapeutic approach that might overcome all of these hurdles. Fusion of transplanted HSPCs might be used to deliver a specific combination of key factors to the targeted cells, once shown to be efficient for inducing conversion of MG into functional photoreceptors. Alternatively, HSPCs can deliver WT alleles after fusion with MG carrying recessive mutations, therefore providing a transversal strategy to correct genetic defects in a variety of patients. HSPCs have also been shown to stabilize retinal blood vessels in $r d 10$ mice, thereby providing a neurotrophic beneficial effect for the preservation of the retinal nuclear layers (53). Importantly, clinical trials have already shown the safety of HSPCs (54).

Still, some questions remain open in our studies. We observed some single-color hybrids (only $\mathrm{YFP}^{+}$), which indicates silencing of the RFP transgene or ploidy reduction. We previously showed that in vivo-formed hybrids appear as synkaryons or heterokaryons, thereby containing 1 or 2 nuclei (28). Multipolar mitosis with ploidy reduction of hybrids was seen in the liver after fusion of hepatocytes with BMDCs (55). On the other hand, heterokaryons have been found in the CNS after BM transplantation (56), which indicates that neurons can cope with high ploidy content.

Here, we also provided evidence that Wnt activation increases the proliferation potential and survival of the hybrids, which in turn downregulate the 2 cell-cycle regulators $\mathrm{p} 27^{\mathrm{Kip} 1}$ and $\mathrm{p} 57^{\mathrm{Kip} 2}$. These 2 factors control retinal progenitor cell-cycle exit and differentiation during retinal development (14). Interestingly, p27 ${ }^{\text {Kip1 }}$ is downregulated very early in MG in regenerating fish retinas (57).
We previously showed that upon retinal injury, MG can fuse with endogenous mobilized BMDCs (28), which suggests that this might also be a physiological response to the damage in mammals and lower vertebrates.

In conclusion, we have identified a route to reprogramming MG in vivo in order to functionally regenerate photoreceptors in a mouse model of retinitis pigmentosa. This approach can be potentially exploited in the future for the treatment of patients.

\section{Methods}

Animal care and treatment. In this study, we used the following male and female transgenic mice: Vav-Cre (58), CAG-RFP (59), Gfap-Cre (60), R26Y (61), RHO-Cre (32), Calr-Cre (33), and nestin-Cre (62). Mice were purchased from Jackson Laboratory. R26Y mice were a gift of M. Studer (Institute of Biology Valrose, Nice, France). For MNU-induced photoreceptor degeneration, adult recipient mice (8 to 12 weeks old) were treated with an i.p. dose of sterile MNU (60 mg/ $\mathrm{kg}$ body weight; Sigma-Aldrich) 24 hours before cell transplantation. For BrdU tracking of proliferating hybrids, mice were treated with a 2 i.p. dose of BrdU ( $15 \mathrm{mg} / \mathrm{kg}$ ) every 12 hours, with the first pulse at the moment of the cell transplantation. Mice were maintained under a 12-hour light/12-hour dark cycle, with access to food and water ad libitum. For the whole study, animals were randomly assigned to treatment groups. We chose groups of a minimum of 6 mice because that was the minimal number of animals required for reaching statistical significance. General anesthesia was induced when needed with i.p. injections of ketamine $(70 \mathrm{mg} / \mathrm{kg}$, Imagene; Merial) and medetomidine (10 mg/kg, Domitor; Pfizer Animal Health).

HSPC isolation and treatment. Donor adult mice (8 to 12 weeks old) were sacrificed by cervical dislocation, and total BM was flushed out from femurs and tibia. HSPCs ( $\mathrm{Lin}^{-}$) were purified from total BM cells by untouched isolation on a magnetic sorter using the Lineage Depletion Kit (Miltenyi Biotec) according to the manufacturer's protocol. Cells were cultured in StemSpan SFEM medium (STEMCELL Technologies) at a concentration of $1 \times 10^{6}$ cells $/ \mathrm{ml}$ in the presence of 100 $\mathrm{ng} / \mathrm{ml}$ recombinant mouse SCF (R\&D Systems), $20 \mathrm{ng} / \mathrm{ml}$ mouse IL-3 (Peprotech), 20 ng/ml human IL-6 (Peprotech), and 10 ng/ml FLT3 ligand (Peprotech). Cells were treated with $1 \mu \mathrm{M}$ BIO (Calbiochem) for 24 hours or untreated as control before transplantation. Thirty minutes before transplantation, cells were incubated with Vybrant DiD Cell-Labeling Solution (Life Technologies) for 30 minutes when necessary, washed twice in PBS, and resuspended to a final concentration of $1 \times 10^{5} \mathrm{cell} / \mu \mathrm{l}$ for transplantation.

Subretinal transplantation of cells. Anesthetized recipient animals received a single $1 \mu \mathrm{l}$ administration of HSPC suspension $\left(1 \times 10^{5}\right.$ cells/ $\mu l)$ by subretinal route. Injections were performed under an operating microscope (Zeiss OPMI Pico; Carl Zeiss Meditec GmbH) with the aid of a 5 - $\mu$ l Hamilton microsyringe (Hamilton Co.). Each mouse was kept on a $37^{\circ} \mathrm{C}$ pad after it was anesthetized, during and after surgery. Briefly, a scalpel was used to make a small cut through the sclera in a temporal position. Then, a 33-gauge bevelled $\left(45^{\circ}\right)$ needle located on a Hamilton syringe system was inserted in the hole between the epithelium and the retina with a tangential angle with the flat part of the bevel toward the retinal side. Cell suspension was injected with a slow and constant pressure. The needle was left in position for another 5 to 10 seconds to allow pressure in the injection system to equilibrate with the eye pressure and then removed by slowly pulling backwards 
to allow the hole in the retina to reseal and to avoid damaging the retina and corneal endothelium during removal.

OCT. To further confirm the correct delivery of the cells in the subretinal space, noninvasive in vivo assessment of retinal detachment formed upon subretinal injections was performed by OCT of 2 groups of mice (group 1, $n=12$; group 2, $n=16$; 4 different litters in total) subretinally injected by 2 different operators. Two independent experiments were sufficient to confirm the same percentage of success of the subretinal injection procedure (32.2\%). For this purpose, groups with different variances were compared. The assessment was performed when mice were under general anesthesia as described in the Animal care and treatment section. Combined confocal scanning laser ophthalmoscopy (cSLO) and SD-OCT imaging were performed using a commercially available device (Spectralis HRA OCT system, Spectralis Eye Explorer 5.6b, Heidelberg Engineering). To adapt the system to the mouse eye, a commercially available $+25 \mathrm{D}$ lens was fit to the system (Heidelberg lens, HE 50744) and the length of the reference pathway was manually adapted according to the manufacturer's instructions. The cSLO system used was an HRA2, using solid source for 488 and $514 \mathrm{~nm}$ and an infrared diode laser for 785 and 815 wavelengths. In order to improve the image acquisition quality, mice wore a PMMA afocal contact lens, with a radius and diameter of $2.7 \mathrm{~mm}$, respectively. Contact lenses were purchased from Cantoor Nissel (Market Place Brackley). Tear substitutive drops were continuously added to improve transparence (Systane, Alcon Labs). Mice were analyzed for cSLO/ SD-OCT with a single scan looking for the injection site.

The correlation analysis between the positive delivery of cells in the subretinal space and the increase in the number of nuclear rows in the ONL, INL, and GC layer (GCL) was carried out blindly before inspection of the histological results.

Electroretinogram tests. For the ERG tests, treated 2-month-old mice were dark adapted for 3 hours, anesthetized, and placed in a stereotaxic apparatus under dim red light. ERGs were evoked by flashes of different light intensities ranging from $10^{-4}$ to $20 \mathrm{~cd} \mathrm{~m}^{-2} \mathrm{~s}^{-1}$ generated through a Ganzfeld stimulator (Lace Elettronica). Three different responses evoked by light were averaged for each luminance step to minimize the noise. The electrophysiological signals were recorded through gold plate electrodes inserted under the lower eyelids referred to a needle electrode inserted s.c. at the level of the corresponding frontal region. The different electrodes were connected to a 2-channel amplifier. The amplitudes of the A- and B-waves measured for each eye upon stimulus with the higher intensity flash under scotopic condition were plotted.

Hybrid isolation for gene expression and flow cytometry analysis. For FACS analysis, retinal samples were isolated, disaggregated in trypsin by mechanical trituration, and filtered. Samples were analyzed by flow cytometry in an LSR I instrument or LSR Fortessa (BD) with FlowJo (Tree Star Inc.) or FACSDiva (BD) software in order to assess the percentage of $\mathrm{DID}^{+} \mathrm{YFP}^{+}$hybrids. In all samples, DAPI (Sigma-Aldrich) was added to exclude dead cells from the analysis.

For gene expression analysis, a BD FACSAria II sorting machine (BD) was used to isolate either the double $\mathrm{DiD}^{+} \mathrm{YFP}^{+}$or $\mathrm{RFP}^{+} \mathrm{YFP}^{+}$ hybrids. DAPI (Sigma-Aldrich) was added to exclude dead cells. Total RNA was extracted using RNA Isolation Mini or Micro Kits (QIAGEN) according to the manufacturer's protocol. The eluted RNA was reverse-transcribed with SuperScript III (Invitrogen), and real-time quantitative PCR (qPCR) reactions using Platinum SYBR
Green qPCix-UDG (Invitrogen) were performed in an LightCycler 480 (Roche) real-time PCR machine according to the manufacturer's recommendations. The oligos used are listed in Supplemental Table 1. Runx1 and Gata1 oligos were purchased from QIAGEN. All of the experiments were performed in triplicate, and differences in cDNA input were compensated by normalizing to the expression of Gapdh. Relative mRNA levels were evaluated as the fold changes with respect to the untreated control or to retinal samples depleted by sorting of either the double $\mathrm{DiD}^{+} \mathrm{YFP}^{+}$or $\mathrm{RFP}^{+} \mathrm{YFP}^{+}$hybrid population.

Fixing, sectioning, immunohistochemistry, and Western blotting. Eyes were fixed by immersion in $4 \%$ paraformaldehyde overnight and then embedded in paraffin. Serial transversal sections of $10-\mu \mathrm{m}$ thickness were processed for TUNEL staining (In Situ Cell Death Detection Kit, Roche) according to the manufacturer's protocol. For immunostaining, sections were treated with serial washing in Xilene for 2 minutes, EtOH 100\% for 1 minute, EtOH 95\% for 1 minute, EtOH 90 for 1 minute, $\mathrm{EtOH} 80 \%$ for 1 minute, $\mathrm{EtOH} 70 \%$ for 1 minute, $\mathrm{EtOH}$ $50 \%$ for 1 minute and $\mathrm{H}_{2} \mathrm{O}$. Slices were placed in a plastic rack with an antigen retrieval buffer (NaCitrate $0.1 \mathrm{M}$ Triton X-100 0.1\%) and boiled for 4 minutes in a domestic microwave oven. After a wash with cold water, sections were blocked in NGS $10 \%$ for 30 minutes and NGS $1 \%$ for 30 minutes. Primary antibodies were incubated in PBS overnight at $4^{\circ} \mathrm{C}$. The primary antibodies used were as follows: rabbit anti-glutamine synthetase GS (1:300, Sigma-Aldrich, G-2781) mouse anti-PCNA (1:200, Sigma-Aldrich, P8825), chicken anti-GFP (1:500, Abcam, AB13970), rabbit anti-recoverin (1:500, Millipore, AB5585), mouse anti-BrdU (1:200, Sigma-Aldrich, B8434), rabbit anti-OTX2 (Abcam, AB21990), rabbit anti-calbindin D-28K (1:50, Sigma-Aldrich, C7354), mouse anti-calretinin (1:100, Millipore, MAB1568), mouse anti-SMI-32 (1:100, Millipore, NE1023), rabbit anti-PKC- $\alpha$ (1:200, Abcam, AB32376), and mouse anti- $\beta$-tubulin III (clone TU-20, Millipore, MAB1637). The secondary antibodies used were as follows: anti-chicken Alexa Fluor 488 (1:1000; Molecular Probes, Invitrogen), anti-chicken Alexa Fluor 568 (1:1000; Molecular Probes, Invitrogen), anti-mouse Alexa Fluor 568 (1:1000; Molecular Probes, A11031, Invitrogen), anti-rabbit Alexa Fluor 568 (1:1000; Molecular Probes, A11036, Invitrogen), anti-mouse Alexa Fluor 633 (1:1000; Molecular Probes, A21050, Invitrogen), and anti-rabbit Alexa Fluor 633 (1:1000; Molecular Probes, A21070, Invitrogen). Nuclei were counterstained with DAPI (Vectashield, Vector Laboratories). Photographs were taken using either an Axioplan microscope (Zeiss) or a Leica laser SP5 or SPE confocal microscopy systems.

For Western blotting, whole cell lysates were prepared by resuspending retinas in $20 \mathrm{mM}$ Tris- $\mathrm{HCl}, \mathrm{pH} 7.5,150 \mathrm{mM} \mathrm{NaCl}, 1 \mathrm{mM}$ $\mathrm{MgCl}_{2}, 0.1 \mathrm{mM} \mathrm{CaCl}, 10 \%$ glycerol, $0.1 \%$ Triton $\mathrm{X}-100$, and protease inhibitors (phenylmethylsulfonyl fluoride and cOmplete protease inhibitor cocktail; Roche Molecular Biochemicals), followed by syringing through a 23-gauge needle. Cell lysates (30 $\mu \mathrm{g} / \mathrm{lane})$ were electrophoresed through a $10 \%$ polyacrylamide-SDS gel and proteins transferred to a polyvinylidene difluoride (PVDF) membrane. Membranes were immunostained overnight at $4^{\circ} \mathrm{C}$ with primary antiPDE6 $\beta$ (1:500, Abcam AB5663) and anti- $\beta$-actin (1:5,000, Abcam $\mathrm{AB} 8226)$ antibodies and 1 hour at room temperature with secondary anti-mouse IgG (1:2000, GE HealthCare, NA931V) and anti-rabbit IgG (1:2000, GE HealthCare, NA934V) antibodies. The signal was visualized using Pierce ECL Western Blotting Substrate (Thermo Scientific) chemiluminescence detection systems. 
Statistics. Percentages of PCNA/YFP or TUNEL/YFP double-positive cells relative to total $\mathrm{YFP}^{+}$detected in the retinas as well as the percentage of YFP/BrdU/marker-positive hybrids relative to the total $\mathrm{YFP} / \mathrm{BrdU}$ cells detected in the retinas were evaluated by staining the tissue sections with anti-GFP and different antibodies or TUNEL and analyzing photographs acquired with $\times 40$ magnification. At least 3 different retinal sections for each eye in 3 different independent experiments were evaluated and results were reported as mean \pm SD. Experiments performed in the whole work with a higher number of duplicates are specified in the text. For the counting of the number of nuclear rows in either the ONL or in the INL and of number of nuclei in the GCL, photographs of the temporal, central, and nasal areas of 9 different serial sections for each eye were acquired with a $\times 40$ magnification and analyzed. Differences were examined using a 2-tailed unpaired Student's $t$ tests or, in the case of multiple comparisons, using 2-way ANOVA test and Bonferroni's post-test. $P<0.05$ was considered significant. The distribution and variance were normal and similar in all groups statistically compared.

Study approval. All of the procedures on mice were reviewed and approved by the Comité Ético de Experimentación Animal del Parc de Recerca Biomèdica de Barcelona (Spain) and were performed according to the ARVO Statement for the Use of Animals in Ophthalmic and Vision Research.

\section{Author contributions}

DS designed and performed the experiments, analyzed data, and wrote the paper. GS performed experiments and analyzed data. UDV performed ERG. NR performed cell sorting. IP performed the OCT analysis on a group of mice. MN supervised the OCT analysis and performed the quality control for the experiments on the rescue of the $r d 10$ phenotype, which were carried out blind. MPC analyzed the data, wrote the paper, and supervised the project.

\section{Acknowledgments}

We would like to thank Andrés Fernández Garcia for support and critical discussions, Francesco Sottile and Ilda Theka for critical reading of the manuscript, the Histological and Microscopy Facilities of CRG and BLOSS Group Company technicians for assistance in the OCT analysis. This work was supported by an ERC grant (242630-RERE to M.P. Cosma), the Ministerio de Economia y Competitividad y FEDER (SAF2011-28580, and BFU2015-71984, BFU2015-71984-ERC to M.P. Cosma), an AGAUR grant from Secretaria d'Universitats i Investigació del Departament d'Economia i Coneixement de la Generalitat de Catalunya (2014SGR1137 to M.P. Cosma), 976_Velux Stiftung (to M.P. Cosma), TV3'12_120530_FUNDACIO MARATO TV3, Ferrer International (to M.P. Cosma and D. Sanges), and Subprograma Juan de la Cierva - Ministerio Ciencia e Innovación (to D. Sanges).

Address correspondence to: Maria Pia Cosma, Center for Genomic Regulation (CRG), C/Dr.Aiguader, 88, 08003 Barcelona, Spain. Phone: 34.9.33160370; E-mail:pia.cosma@crg.es.
1. Blau HM, et al. Plasticity of the differentiated state. Science. $1985 ; 230(4727): 758-766$.

2. Gurdon JB, Elsdale TR, Fischberg M. Sexually mature individuals of Xenopus laevis from the transplantation of single somatic nuclei. Nature. 1958;182(4627):64-65

3. Takahashi K, Ichisaka T, Yamanaka S. Identification of genes involved in tumor-like properties of embryonic stem cells. Methods Mol Biol. 2006;329:449-458.

4. Jaenisch R, Young R. Stem cells, the molecular circuitry of pluripotency and nuclear reprogramming. Cell. 2008;132(4):567-582.

5. Sanges D, Cosma MP. Reprogramming cell fate to pluripotency: the decision-making signalling pathways. Int J Dev Biol. 2010;54(11-12):1575-1587.

6. Arlotta P, Berninger B. Brains in metamorphosis: reprogramming cell identity within the central nervous system. Curr Opin Neurobiol. 2014;27:208-214.

7. Torper O, et al. Generation of induced neurons via direct conversion in-vivo. Proc Natl Acad Sci US A. 2013;110(17):7038-7043.

8. Niu W, et al. In vivo reprogramming of astrocytes to neuroblasts in the adult brain. Nat Cell Biol. 2013;15(10):1164-1175

9. Reichenbach A, Bringmann A. New functions of Muller cells. Glia. 2013;61(5):651-678.

10. Fausett BV, Goldman D. A role for $\alpha 1$ tubulinexpressing Muller glia in regeneration of the injured zebrafish retina. J Neurosci. 2006;26(23):6303-6313.

11. Ramachandran R, Fausett BV, Goldman D. Ascl1a regulates Muller glia dedifferentiation and ret- inal regeneration through a Lin-28-dependent, let-7 microRNA signalling pathway. Nat Cell Biol. 2010;12(11):1101-1107.

12. Powell C, Grant AR, Cornblath E, Goldman D. Analysis of DNA methylation reveals a partial reprogramming of the Muller glia genome during retina regeneration. Proc Natl Acad Sci U S A. 2013;110(49):19814-19819.

13. Bringmann A, et al. Role of retinal glial cells in neurotransmitter uptake and metabolism. Neurochem Int. 2009;54(3-4):143-160.

14. Dyer MA, Cepko CL. Control of Muller glial cell proliferation and activation following retinal injury. Nat Neurosci. 2000;3(9):873-880.

15. Ooto $S$, et al. Potential for neural regeneration after neurotoxic injury in the adult mammalian retina. Proc Natl Acad Sci U S A. 2004;101(37):13654-13659.

16. Osakada F, Ooto S, Akagi T, Mandai M, Akaike A, Takahashi M. Wnt signaling promotes regeneration in the retina of adult mammals. J Neurosci. 2007;27(15):4210-4219.

17. Pollak J, et al. ASCL1 reprograms mouse Muller glia into neurogenic retinal progenitors. Development. 2013;140(12):2619-2631.

18. Goldman D. Muller glial cell reprogramming and retina regeneration. Nat Rev Neurosci. 2014;15(7):431-442.

19. Karl MO, Hayes S, Nelson BR, Tan K, Buckingham B, Reh TA. Stimulation of neural regeneration in the mouse retina. Proc Natl Acad Sci US A. 2008;105(49):19508-19513.

20. Lluis F, Pedone E, Pepe S, Cosma MP. Periodic activation of Wnt/beta-catenin signaling enhances somatic cell reprogramming mediated by cell fusion. Cell Stem Cell. 2008;3(5):493-507.

21. Lluis F, Ombrato L, Pedone E, Pepe S, Merrill

BJ, Cosma MP. T-cell factor 3 (Tcf3) deletion increases somatic cell reprogramming by inducing epigenome modifications. Proc Natl Acad Sci U S A. 2011;108(29):11912-11917.

22. Ferrari G, et al. Muscle regeneration by bone marrow-derived myogenic progenitors. Science. 1998;279(5356):1528-1530

23. Petersen BE, et al. Bone marrow as a potential source of hepatic oval cells. Science. 1999;284(5417):1168-1170.

24. Mezey E, Chandross KJ, Harta G, Maki RA, McKercher SR. Turning blood into brain: cells bearing neuronal antigens generated in-vivo from bone marrow. Science. 2000;290(5497):1779-1782.

25. Johansson CB, et al. Extensive fusion of haematopoietic cells with Purkinje neurons in response to chronic inflammation. Nat Cell Biol. 2008;10(5):575-583.

26. Krause DS, et al. Multi-organ, multi-lineage engraftment by a single bone marrow-derived stem cell. Cell. 2001;105(3):369-377.

27. Sanges D, Lluis F, Cosma MP. Cell-fusionmediated reprogramming: pluripotency or trans differentiation? Implications for regenerative medicine. Adv Exp Med Biol. 2011;713:137-159.

28 . Sanges $\mathrm{D}$, et al. Wnt/ $\beta$-catenin signaling triggers neuron reprogramming and regeneration in the mouse retina. Cell Rep. 2013;4(2):271-286.

29. Su M, Hu H, Lee Y, d’Azzo A, Messing A, Brenner M. Expression specificity of GFAP transgenes. 
Neurochem Res. 2004;29(11):2075-2093.

30. Ogino $\mathrm{H}$, et al. Retinal degeneration induced by $\mathrm{N}$-methyl-N-nitrosourea and detection of 7-methyldeoxyguanosine in the rat retina. Toxicol Pathol. 1993;21(1):21-25.

31. Sanges D, Comitato A, Tammaro R, Marigo V. Apoptosis in retinal degeneration involves crosstalk between apoptosis-inducing factor (AIF) and caspase-12 and is blocked by calpain inhibitors. Proc Natl Acad Sci U S A. 2006;103(46):17366-17371.

32. Li S, Chen D, Sauve Y, McCandless J, Chen YJ, Chen CK. Rhodopsin-iCre transgenic mouse line for Cre-mediated rod-specific gene targeting. Genesis. 2005;41(2):73-80.

33. Haverkamp S, Inta D, Monyer H, Wassle H. Expression analysis of green fluorescent protein in retinal neurons of four transgenic mouse lines. Neuroscience. 2009;160(1):126-139.

34. Fantl V, Stamp G, Andrews A, Rosewell I, Dickson C. Mice lacking cyclin D1 are small and show defects in eye and mammary gland development. Genes Dev. 1995;9(19):2364-2372.

35. Ma C, Papermaster D, Cepko CL. A unique pattern of photoreceptor degeneration in cyclin D1 mutant mice. Proc Natl Acad Sci U S A. 1998;95(17):9938-9943.

36. Ma TC, et al. A screen for inducers of p21(waf1/ cip1) identifies HIF prolyl hydroxylase inhibitors as neuroprotective agents with antitumor properties. Neurobiol Dis. 2013;49:13-21.

37. Levine EM, Close J, Fero M, Ostrovsky A, Reh TA. p27(Kip1) regulates cell cycle withdrawal of late multipotent progenitor cells in the mammalian retina. Dev Biol. 2000;219(2):299-314.

38. Schreiber D, Janisch W, Warzok R, Tausch H. [Induction of brain and spinal cord tumors in rabbits with N-methyl-N-nitrosourea]. Z Gesamte Exp Med. 1969;150(1):76-86.

39. Rosch S, Johnen S, Mataruga A, Muller F, Pfarrer C, Walter P. Selective photoreceptor degeneration by intravitreal injection of N-methyl$\mathrm{N}$-nitrosourea. Invest Ophthalmol Vis Sci. 2014;55(3):1711-1723.

40. Gargini C, Terzibasi E, Mazzoni F, Strettoi E. Ret- inal organization in the retinal degeneration 10 (rd10) mutant mouse: a morphological and ERG study. J Comp Neurol. 2007;500(2):222-238.

41. Tomita T, Funaishi A. Studies on intraretinal action potential with low-resistance microelectrode. J Neurophysiol. 1952;15(1):75-84.

42. Penn RD, Hagins WA. Signal transmission along retinal rods and the origin of the electroretinographic a-wave. Nature. 1969;223(5202):201-204.

43. McLaughlin ME, Ehrhart TL, Berson EL, Dryja TP. Mutation spectrum of the gene encoding the beta subunit of rod phosphodiesterase among patients with autosomal recessive retinitis pigmentosa. Proc Natl Acad Sci U S A. 1995;92(8):3249-3253.

44. Danciger $\mathrm{M}$, et al. Mutations in the PDE6B gene in autosomal recessive retinitis pigmentosa. Genomics. 1995;30(1):1-7.

45. Byrne LC, et al. Viral-mediated RdCVF and RdCVFL expression protects cone and rod photoreceptors in retinal degeneration.J J Clin Invest. 2015;125(1):105-116.

46. Kolomeyer AM, Zarbin MA. Trophic factors in the pathogenesis and therapy for retinal degenerative diseases. Surv Ophthalmol. 2014;59(2):134-165.

47. Egensperger R, Maslim J, Bisti S, Holländer H, Stone J. Fate of DNA from retinal cells dying during development: uptake by microglia and macroglia (Müller cells). Brain Res Dev Brain Res. 1996;97(1):1-8.

48. Morris AC, Schroeter EH, Bilotta J, Wong RO, Fadool JM. Cone survival despite rod degeneration in XOPS-mCFP transgenic zebrafish. Invest Ophthalmol Vis Sci. 2005;46(12):4762-4771.

49. Bailey TJ, Fossum SL, Fimbel SM, Montgomery JE, Hyde DR. The inhibitor of phagocytosis, O-phospho-L-serine, suppresses Müller glia proliferation and cone cell regeneration in the light-damaged zebrafish retina. Exp Eye Res. 2010;91(5):601-612.

50 . Wehner $\mathrm{D}$, et al. Wnt $/ \beta$-catenin signaling defines organizing centers that orchestrate growth and differentiation of the regenerating zebrafish caudal fin. Cell Rep. 2014;6(3):467-481.

51. Lin G, Slack JM. Requirement for Wnt and FGF signaling in Xenopus tadpole tail regeneration.
Dev Biol. 2008;316(2):323-335.

52. Braisted JE, Essman TF, Raymond PA. Selective regeneration of photoreceptors in goldfish retina. Development. 1994;120(9):2409-2419.

53. Otani A, et al. Rescue of retinal degeneration by intravitreally injected adult bone marrow-derived lineage-negative hematopoietic stem cells. JClin Invest. 2004;114(6):765-774.

54. Park SS, et al. Intravitreal autologous bone marrow $\mathrm{CD} 34^{+}$cell therapy for ischemic and degenerative retinal disorders: preliminary phase 1 clinical trial findings. Invest Ophthalmol Vis Sci. 2015;56(1):81-89.

55. Duncan AW, et al. Ploidy reductions in murine fusion-derived hepatocytes. PLoS Genet. 2009;5(2):e1000385.

56. Weimann JM, Johansson CB, Trejo A, Blau HM. Stable reprogrammed heterokaryons form spontaneously in Purkinje neurons after bone marrow transplant. Nat Cell Biol. 2003;5(11):959-966.

57. Kassen SC, et al. Time course analysis of gene expression during light-induced photoreceptor cell death and regeneration in albino zebrafish. Dev Neurobiol. 2007;67(8):1009-1031.

58. Stadtfeld M, Graf T. Assessing the role of hematopoietic plasticity for endothelial and hepatocyte development by non-invasive lineage tracing. Development. 2005;132(1):203-213.

59. Long JZ, Lackan CS, Hadjantonakis AK. Genetic and spectrally distinct in vivo imaging: embryonic stem cells and mice with widespread expression of a monomeric red fluorescent protein. BMC Biotechnol. 2005;5:20.

60. Zhuo L, Theis M, Alvarez-Maya I, Brenner M, Willecke K, Messing A. hGFAP-cre transgenic mice for manipulation of glial and neuronal function in-vivo. Genesis. 2001;31(2):85-94.

61. Srinivas S, et al. Cre reporter strains produced by targeted insertion of EYFP and ECFP into the ROSA26 locus. BMC Dev Biol. 2001;1:4.

62. Kellendonk C, Tronche F, Casanova E, Anlag K, Opherk C, Schutz G. Inducible site-specific recombination in the brain. J Mol Biol. 1999;285(1):175-182. 